\title{
Natural convection of Bingham fluids in rectangular cross-sectional cylindrical annuli with differentially heated vertical walls
}

Sahin Yigit $^{1, \bowtie}$, Nilanjan Chakraborty ${ }^{1}$

${ }^{1}$ School of Engineering, Newcastle University, Newcastle upon Tyne NE1 7RU, UK

$凶$ corresponding author.

s.yigit1@ncl.ac.uk

KEYWORDS: natural convection, cylindrical annular enclosure, aspect ratio, yield stress fluids, Bingham model. 


\section{ABSTRACT}

Natural convection of yield stress fluids in rectangular cross-sectional cylindrical annular enclosures has been numerically analysed in this study. The laminar steady-state simulations have been conducted for a range of different values of normalised internal radius $\left(r_{i} / L\right.$ from $1 / 8$ to 16 , where $L$ is the difference between outer and inner radii); aspect ratios $(A R=H / L$ from $1 / 8$ to 8 where $H$ is the enclosure height); and nominal Rayleigh number ( $R a$ from $10^{3}$ to $10^{6}$ ) for a single representative value of Prandtl number $(\mathrm{Pr}$ is 500). The Bingham model has been used to mimic the yield stress fluid motion, and numerical simulations have been conducted for both constant wall temperature (CWT) and constant wall heat flux (CWHF) boundary conditions for the vertical side walls. It is found that the mean Nusselt number based on the inner periphery $\overline{N u}_{i}$ increases (decreases) with an increase in $R a(B n)$ due to augmented buoyancy (viscous) forces irrespective of the boundary condition. The ratio of convective to diffusive thermal transport increases with increasing $r_{i} / L$ for both Newtonian (i.e. $B n=0$ ) and Bingham fluids regardless of the boundary condition. Moreover, the mean Nusselt number $\overline{N u}_{i}$ normalised by the corresponding Nusselt number due to pure conductive transport (i.e. $\overline{N u}_{i} / \overline{N u}$ cond ) shows a nonmonotonic trend with increasing $A R$ in the CWT configuration for a given set of values of $R a, P r, r_{i} / L$ for both Newtonian (i.e. $B n=0$ ) and Bingham fluids, whereas $\overline{N u}_{i} / \overline{N u}_{c o n d}$ increases monotonically with increasing $A R$ in the CWHF configuration. The influences of convective thermal transport strengthen while thermal diffusive transport weakens with increasing $A R$, and these competing effects are responsible for the non-monotonic $\overline{N u}_{i} / \overline{N u}_{\text {cond }}$ variation with $A R$ in the CWT configuration. Detailed scaling analysis is utilised to explain the observed influences of $R a, B n, r_{i} / L$ and $A R$, which along with the simulation data has been utilised to propose correlations for $\overline{N u}_{i}$. 


\section{NOMENCLATURE}

\begin{tabular}{|c|c|}
\hline$A U R s$ & Apparently Unyielded Regions \\
\hline$A R$ & Aspect Ratio $(H / L)$, dimensionless \\
\hline$b_{0-3}$ & Correlation parameter, dimensionless \\
\hline$B n$ & Bingham number, dimensionless \\
\hline$B n_{c}$ & Critical Bingham number, dimensionless \\
\hline$B n_{\max }$ & $\begin{array}{l}\text { Threshold value of Bingham number above which } \\
\overline{N u}_{i} /\left(\overline{N u}_{i}\right)_{\text {cond }} \text { settles to unity, dimensionless }\end{array}$ \\
\hline$B n_{\max }{ }^{*}$ & $\begin{array}{l}\text { Scaled threshold value of Bingham number, } \\
\text { dimensionless }\end{array}$ \\
\hline$c_{1}, c_{2}$ & Correlation parameter, dimensionless \\
\hline CWT & Constant wall temperature \\
\hline CWHF & Constant wall heat flux \\
\hline$d_{0-7}$ & Correlation parameter, dimensionless \\
\hline$f_{1}, f_{2}, f_{3}$ & Functions, dimensionless \\
\hline$g$ & Gravitational acceleration, $\mathrm{m} / \mathrm{s}^{2}$ \\
\hline$G r$ & Grashof number, dimensionless \\
\hline$h$ & Heat transfer coefficient, $\mathrm{W} / \mathrm{m}^{2} \mathrm{~K}$ \\
\hline$H$ & Height of the enclosure, $\mathrm{m}$ \\
\hline$k$ & Thermal conductivity, $\mathrm{W} / \mathrm{mK}$ \\
\hline$k_{0-6}$ & Correlation parameter, dimensionless \\
\hline$L$ & Difference between inner and outer radius, $m$ \\
\hline$m$ & Stress growth exponent, $\mathrm{s}$ \\
\hline$m_{0-2}$ & Correlation parameter, dimensionless \\
\hline$N u$ & Nusselt number, dimensionless \\
\hline$n_{1-3}$ & Correlation parameter, dimensionless \\
\hline$P$ & Pressure, $\mathrm{Pa}$ \\
\hline $\operatorname{Pr}$ & Prandtl number, dimensionless \\
\hline$q$ & Heat flux, W/m² \\
\hline$r$ & Radial coordinate, $\mathrm{m}$ \\
\hline$r_{i}$ & Inner radius, $m$ \\
\hline$r_{o}$ & Outer radius, $\mathrm{m}$ \\
\hline$R^{2}$ & Coefficient of determination, dimensionless \\
\hline$R a$ & Rayleigh number, dimensionless \\
\hline$T$ & Temperature, $\mathrm{K}$ \\
\hline$T_{C}$ & Cold wall temperature, $\mathrm{K}$ \\
\hline$T_{H}$ & Hot wall temperature, $\mathrm{K}$ \\
\hline$u$ & Radial velocity component, m/s \\
\hline$U$ & $\begin{array}{l}\text { Dimensionless radial velocity }(U=u L / \alpha) \text {, } \\
\text { dimensionless }\end{array}$ \\
\hline$U_{\text {ref }}$ & Reference velocity scale, $\mathrm{m} / \mathrm{s}$ \\
\hline$w$ & Vertical velocity component, $\mathrm{m} / \mathrm{s}$ \\
\hline$W$ & $\begin{array}{l}\text { Dimensionless vertical velocity }(U=w L / \alpha) \text {, } \\
\text { dimensionless }\end{array}$ \\
\hline$z$ & Coordinate in vertical direction, $\mathrm{m}$ \\
\hline \multicolumn{2}{|c|}{ Greek Symbols } \\
\hline$\alpha$ & Thermal diffusivity, $\mathrm{m}^{2} / \mathrm{s}$ \\
\hline$\beta$ & Coefficient of thermal expansion, $1 / \mathrm{K}$ \\
\hline$\delta_{t h}$ & Thermal boundary layer thickness, $\mathrm{m}$ \\
\hline$\delta$ & $\begin{array}{l}\text { Hydro-dynamic boundary layer thickness for vertical } \\
\text { walls, } m\end{array}$ \\
\hline$\delta_{1}$ & $\begin{array}{l}\text { Hydro-dynamic boundary layer thickness for horizontal } \\
\text { walls, m }\end{array}$ \\
\hline$\theta$ & Dimensionless temperature, dimensionless \\
\hline$\mu$ & Dynamic viscosity, $\mathrm{Ns} / \mathrm{m}^{2}$ \\
\hline$\rho$ & Density, $\mathrm{kg} / \mathrm{m}^{3}$ \\
\hline$\dot{\gamma}$ & Second invariant of strain rate tensor, $\mathrm{s}^{-1}$ \\
\hline$\dot{\gamma}$ & Strain rate tensor, $\mathrm{s}^{-1}$ \\
\hline
\end{tabular}

$\tau \quad$ Second invariant of viscous stress tensor, $\mathrm{Pa}$

$\tau_{i j} \quad$ Components of viscous stress tensor, $\mathrm{Pa}$

$\tau_{y} \quad$ Yield stress, $\mathrm{Pa}$

$\tau_{y} \quad$ Yield stress tensor, $\mathrm{Pa}$

$\underline{\tau} \quad$ Viscous stress tensor, $\mathrm{Pa}$

$\phi \quad$ Azimuthal coordinate, dimensionless

$\psi \quad$ Stream function, $\mathrm{m}^{2} / \mathrm{s}$

$\Psi \quad$ Dimensionless stream function, dimensionless

$\Delta T \quad$ Temperature difference, $\mathrm{K}$

\section{Subscripts}

cen Geometrical centre of the domain

cond Conduction

CWHF Constant wall heat flux

$C W T \quad$ Constant wall temperature

eff Effective value

$\max \quad$ Maximum value

rec Rectangular

ref Reference value

$i \quad$ Inner periphery wall

$o \quad$ Outer periphery wall

\section{Superscripts}

- Mean value

$+\quad$ Non-dimensionalised value

$V \quad$ Representative value in the vertical boundary

$H \quad$ Representative value in the horizontal 


\section{INTRODUCTION}

Natural convection in enclosed spaces has been extensively analysed (see (Catton et al., 1974; Ostrach, 1988) and references therein) in heat transfer literature because of its wide range of applications (e.g. from domestic heating to solar collectors, to name a few). Most existing analyses on natural convection in enclosures have been carried out for Newtonian fluids in rectangular enclosures (Catton et al., 1974; Ostrach, 1988) but relatively limited attention has been directed to natural convection in cylindrical annuli (de Vahl Davis and Thomas, 1969; Keyhani et al., 1983; Nagendra et al., 1970; Satya Sai et al., 1993 ) in spite of its greater relevance to engineering applications than rectangular enclosures.

Most man-made fluids are non-Newtonian in nature (i.e. viscosity is not a constant and dependent on the shear rate at a given temperature), and yield stress fluid is one of the categories of non-Newtonian fluids which does not flow until a threshold stress (i.e. yield stress) is surpassed. In the recent past, a number of analyses (Balmforth and Rust, 2009; Darbouli et al., 2013; Hassan et al., 2013, 2015; Kebiche et al., 2014; Turan et al., 2010, 2012a; Turan et al., 2011a, 2012b, 2014; Turan et al., 2011b; Turan et al., 2017; Vikhansky, 2009, 2010; Yigit and Chakraborty, 2017a, b; Yigit et al., 2016; Yigit et al., 2017; Yigit et al., 2015a, b; Zhang et al., 2006) concentrated on natural convection of yield stress fluids and the main findings of these studies are summarised in Table 1. It can be seen from Table 1 that most of these analyses were conducted for the Bingham model, which is the simplest form of yield stress fluids and shows a linear strain rate dependence of viscous stress. Furthermore, the description of Table 1 reveals that the investigations on natural convection of yield stress fluids can be divided in two broad categories. The first category concentrated on the critical condition under which the fluid flow is initiated (e.g. (Balmforth and Rust, 2009; Darbouli et al., 2013; Kebiche et al., 2014; Turan et al., 2017; Vikhansky, 2009, 2010; Zhang et al., 2006), whereas the second category deals with heat transfer characteristics beyond the critical condition for flow initiation where the effects of convective transport are well-established (e.g. (Hassan et al., 2013, 2015; Turan et al., 2010, 2012a; Turan et al., 2011a, 2012b, 2014; Turan et al., 2011b; Yigit and Chakraborty, 2017a, b; Yigit et al., 2016; Yigit et al., 2017; Yigit et al., 2015a, b). The analyses belonging to the second category demonstrated weakening of convective transport as a result of increased flow resistance arising from the yield stress.

Furthermore, all the analyses apart from the studies by (Yigit and Chakraborty, 2017b; Yigit et al., 2016; Yigit et al., 2017) were carried out for rectangular enclosures and among them (Turan et al., 2011a, 2014; Yigit and Chakraborty, 2017a; Yigit et al., 2015a) indicated that the aspect ratio (height: length) significantly affects the heat transfer characteristics in natural convection of both Newtonian and Bingham fluids in rectangular enclosures. For example, the convective (diffusive) transport strengthens (weakens) with increasing aspect ratio $A R$ for differentially heated vertical sidewalls (Turan et al., 2011a, 2014), whereas an opposite behaviour is observed for Rayleigh-Bénard convection (Yigit and Chakraborty, 2017a; Yigit et al., 2015a). However, the influence of aspect ratio (height: distance between outer and inner radii) in vertical cylindrical annuli with differentially heated vertical walls is yet to be analysed in the existing literature and this behaviour is expected to be different to that in 
rectangular enclosures due to the influences of wall curvature. This gap in the existing literature is addressed here by numerically analysing natural convection of Bingham fluids in vertical cylindrical annuli with differentially heated vertical walls for different nominal Rayleigh number values given by $10^{3} \leq R a \leq 10^{6}$, aspect ratios within the range given by $1 / 8 \leq A R \leq 8$ (i.e. $A R=H / L$ where $H$ is the enclosure height and $L$ is the difference between outer and inner radii) and normalised inner radii $r_{i} / L$ ranging from 0.125 to 16 for a nominal representative Prandtl number $\operatorname{Pr}=500$. The current analyses have been carried out for a single representative value of nominal Prandtl number (i.e. $\operatorname{Pr}=$ $\mu c_{p} / k=500$ ) since practical yield stress fluids exhibit Prandtl numbers of the order of 100 (Darbouli et al., 2013; Hassan et al., 2015; Kebiche et al., 2014). For example, a recent experimental analysis (Kebiche et al., 2014) based on experimental analysis of Rayleigh-Bénard convection of yield stress fluids in rectangular enclosures reported that $0.05 \%$ (by mass) Carpool solution in water shows a yield stress behaviour with a nominal Prandtl number of $\operatorname{Pr} \approx 350$.

It was demonstrated in several previous analyses (Turan et al., 2012b, 2014; Turan et al., 2011b; Yigit and Chakraborty, 2017a) that the boundary condition of the differentially heated side walls significantly affects the aspect ratio $A R$ dependence of the mean Nusselt number $\overline{N u}$ in rectangular enclosures, and thus both constant wall temperature (CWT) and constant wall heat flux (CWHF) boundary conditions have been considered for this analysis. In this respect, the main objectives of this analysis are:

1. To analyse the influences of $A R$ on $R a$ and $r_{i} / L$ dependences of the mean Nusselt number $\overline{N u}$ for natural convection of Bingham fluids in cylindrical annular spaces with differentially heated vertical walls.

2. To provide physical explanations for the above influences using scaling arguments, and parameterise the numerical findings in the form of correlations for the mean Nusselt number $\overline{N u}$.

The rest of the paper will be organised as follows. The mathematical background and numerical implementation related information will be provided in the next two sub-sections. This will be followed by a detailed scaling analysis. Following this, the results will be discussed, and finally conclusions will be drawn.

\section{MATHEMATICAL BACKGROUND}

The strain rate dependence of viscous stresses according to the Bingham model (Barnes, 1999) is expressed as:

$$
\begin{array}{lll}
\underline{\underline{\gamma}}=0 & \text { for } & \tau \leq \tau_{y}, \\
\underline{\underline{\tau}}=\left(\mu+\tau_{y} / \dot{\gamma}\right) \underline{\underline{\dot{\gamma}}} & \text { for } & \tau>\tau_{y},
\end{array}
$$


where the components of the rate of strain tensor $\underline{\underline{\underline{\gamma}}}$ are given by: $\dot{\gamma}_{i j}=\left(\partial u_{i} / \partial x_{j}+\partial u_{j} / \partial x_{i}\right), \underline{\underline{\tau}}$ is the stress tensor, $\tau_{y}$ is the yield stress, $\mu$ is the plastic viscosity. In Eqs. (1) and (2), $\tau$ and $\dot{\gamma}$ are the second invariants of the stress and the rate of strain tensors respectively, which are expressed as:

$$
\begin{aligned}
& \tau=\left[\frac{1}{2} \underline{\underline{\tau}}: \underline{\underline{\tau}}\right]^{1 / 2}, \\
& \dot{\gamma}=\left[\frac{1}{2} \underline{\underline{\gamma}}: \dot{\underline{\gamma}}\right]^{1 / 2} .
\end{aligned}
$$

Here, the bi-viscosity regularisation (O'Donovan and Tanner, 1984) is used to model the stress-shear rate characteristics for Bingham fluids:

$\underline{\underline{\tau}}=\mu_{\text {yield }} \underline{\underline{\dot{\gamma}}} \quad$ for $\quad \dot{\gamma} \leq \tau_{y} / \mu_{\text {yield }}$

$\underline{\underline{\tau}}=\tau_{y}(\underline{\underline{\gamma}} / \dot{\gamma})+\mu \underline{\underline{\gamma}} \quad$ for $\quad \dot{\gamma}>\tau_{y} / \mu_{y i e l d}$

where $\mu_{\text {yield }}$ is the yield viscosity. O'Donovan and Tanner (1984) demonstrated that a value of $\mu_{\text {yield }} \geq$ $1000 \mu$ mimics the true Bingham model in a satisfactory manner, and accordingly $\mu_{\text {yield }} / \mu=10^{4}$ is taken here. Moreover, a limited number of simulations have also been conducted for Papanastasiou's regularisation (Papanastasiou, 1987), which takes the following form:

$$
\underline{\underline{\tau}}=\underline{\underline{\tau_{y}}}(1-\exp (-m \dot{\gamma}))+\mu \underline{\underline{\underline{\gamma}}}
$$

where $m$ is the stress growth exponent which has the dimension of time (i.e. $m>>10 L^{2} / \alpha$ ). The stress growth exponent has been chosen to be $m>>10^{5} L^{2} / \alpha$ in this analysis to mimic the true Bingham model in a satisfactory manner. Both bi-viscosity and Papanastasiou regularisations (i.e. Eqs. (5) and (6)) convert the "unyielded" region to a zone of high viscosity such that the numerical solutions predict negligible magnitudes of velocity in these regions so fluid flow does not influence the thermal transport, and heat transfer takes place principally due to conduction. The maximum difference between the mean Nusselt numbers obtained from these two regularizations remains within the typical uncertainties encountered in experimental investigations ( 2-3\% shown in Table 1 of (Yigit and Chakraborty, 2017b)). Although more computationally sophisticated but expensive techniques (Huilgol and Kefayati, 2015; Karimfazli et al., 2015) than the regularisation methods can offer higher degree of accuracy in capturing the onset of viscoplastic fluid motion, the current paper focuses principally on convective heat transfer well beyond the critical condition for the onset of fluid movement. The fluid motion becomes too weak to impart any influence on the convective heat transfer for Bingham numbers smaller than the threshold Bingham number value at which fluid flow entirely stops so the regularisation based methods offer computational economy without compromising any physical accuracy for heat transfer problems. The Nusselt number for natural convection of Bingham fluids in rectangular cross-sectional cylindrical annular enclosures can be expressed as: $N u=f_{1}\left(R a, P r, B n, r_{i} / L, A R\right)$ according to Buckingham's $\pi$ 
teorem. The nominal Rayleigh, Prandtl and Bingham (i.e. ratio of yield stress to viscous stress) numbers can be defined for both CWT and CWHF boundary conditions in the following manner:

$R a_{C W T}=\frac{\rho g \beta\left(T_{H}-T_{C}\right) L^{3}}{\mu \alpha}=G r_{C W T} \operatorname{Pr}$ and $B n_{C W T}=\frac{\tau_{y} L}{\mu \sqrt{g \beta\left(T_{H}-T_{C}\right) L}} ;$

$R a_{C W H F}=\frac{\rho g \beta q_{i} L^{4}}{k \mu \alpha}=G r_{C W H F} \operatorname{Pr}$ and $B n_{C W H F}=\frac{\tau_{y}}{\mu \sqrt{g \beta q_{i} / k}}$,

where $G r_{C W T}=\rho^{2} g \beta\left(T_{H}-T_{C}\right) L^{3} / \mu^{2}\left(G r_{C W H F}=\rho^{2} g \beta q_{i} L^{4} / k \mu^{2}\right)$ is the nominal Grashof number for the CWT (CWHF) boundary condition. The local heat transfer coefficient $h_{i}$ for the internal radius can be expressed as:

$h_{i}=\left|-k\left(\frac{\partial T}{\partial r}\right)_{r=r_{i}} \times 1 /\left(T_{r=r_{i}}-T_{r=r_{i}+L}\right)\right|$.

The mean heat transfer coefficient $\bar{h}_{i}$ and the mean Nusselt number $\overline{N u}_{i}$ based on the internal radius are evaluated as:

$\bar{h}_{i}=\frac{1}{H} \int_{0}^{H} h_{i} d z$ and $\overline{N u}_{i}=\frac{\bar{h}_{i} L}{k}$.

According to steady state, one obtains:

$\frac{q_{o}}{q_{i}}=\frac{r_{i}}{r_{o}}$,

where $q_{i}$ and $q_{o}$ are the mean heat flux magnitudes at the internal and external radii respectively, which can be defined as:

$q_{i}=\frac{1}{H} \int_{0}^{H}\left|k \frac{\partial T}{\partial r}\right|_{r=r_{i}} d z$ and $q_{o}=\frac{1}{H} \int_{0}^{H}\left|k \frac{\partial T}{\partial r}\right|_{r=r_{o}} d z$.

This give rise to:

$\bar{h}_{o}=\bar{h}_{i} \frac{r_{i}}{r_{o}}$ and $\overline{N u}_{o}=\overline{N u}_{i} \frac{r_{i}}{r_{o}}$.

According to pure conduction solution, the heat flux on the internal surface can be expressed as:

$\left(q_{i}\right)_{\text {cond }}=\frac{k\left|T_{r=r_{i}}-T_{r=r_{o}}\right|}{r_{i} \ln \left(1+L / r_{i}\right)}$.

Using Eqs. (12) and (13), the Nusselt number due to pure conduction on the internal surface can be expressed as:

$\left(\overline{N u}_{i}\right)_{\text {cond }}=\frac{\left(q_{i}\right)_{c o n d} L}{k\left(T_{H}-T_{C}\right)}=\frac{\left(L / r_{i}\right)}{\ln \left(1+L / r_{i}\right)}$.

The current analysis has been carried out in non-dimensional form for the sake of generalisation. The spatial co-ordinates, velocity components, pressure and temperature can be non-dimensionalised in the following manner:

$r^{+}=r / L ; z^{+}=z / L ; u_{i}^{+}=u_{i} / U_{\text {ref }} ; P^{+}=P / \rho U_{r e f}^{2} ; \Theta=\left(T-T_{\text {ref }}\right) / \Delta T_{\text {ref }}$,

where $U_{\text {ref }}$ is taken to be $\sqrt{g \beta \Delta T_{\text {ref }} L}$ based on the equilibrium of inertial and the buoyancy forces (Hassan et al., 2013, 2015; Turan et al., 2010, 2012a) where $\Delta T_{\text {ref }}$ is a reference temperature difference. For the CWT boundary condition, $\Delta T_{\text {ref }}$ can be taken to be $\Delta T=\left(T_{H}-T_{C}\right)$ while it can be taken to be $\Delta T_{r e f}=$ 
$q_{i} L / k$ for the CWHF configuration. Additionally, the reference temperature is taken to be temperature at the centre of the domain $T_{c e n}$ for the CWHF boundary condition, whereas the cold wall temperature $T_{C}$ is considered to be the reference temperature for the CWT boundary condition. The steady-state nondimensional governing equations for mass, momentum and energy for constant temperatureindependent thermo-physical properties under the assumption of axisymmetry take the following form: Non-dimensional mass conservation equation:

$\frac{1}{r^{+}} \frac{\partial\left(r^{+} u^{+}\right)}{\partial r^{+}}+\frac{\partial w^{+}}{\partial z^{+}}=0$

\section{Non-dimensional momentum conservation equations}

\section{$\underline{\text { Radial direction: }}$}

$u^{+} \frac{\partial u^{+}}{\partial r^{+}}+w^{+} \frac{\partial u^{+}}{\partial z^{+}}=-\frac{\partial P^{+}}{\partial r^{+}}+\frac{P r^{1 / 2}}{R a^{1 / 2}}\left[\frac{1}{r^{+}} \frac{\partial\left(r^{+} \tau_{r r^{+}}\right)}{\partial r^{+}}-\frac{\tau_{\phi \phi^{+}}}{r^{+}}+\frac{\partial\left(\tau_{r z}{ }^{+}\right)}{\partial z^{+}}\right] ;$

Vertical direction:

$u^{+} \frac{\partial w^{+}}{\partial r^{+}}+w^{+} \frac{\partial w^{+}}{\partial z^{+}}=-\frac{\partial P^{+}}{\partial z^{+}}+\Theta+\frac{P r^{1 / 2}}{R a^{1 / 2}}\left[\frac{1}{r^{+}} \frac{\partial\left(r^{+} \tau_{r z}\right)}{\partial r^{+}}+\frac{\partial\left(\tau_{z z}{ }^{+}\right.}{\partial z^{+}}\right]$

Non-dimensional energy conservation equation:

$u^{+} \frac{\partial \Theta}{\partial r^{+}}+w^{+} \frac{\partial \Theta}{\partial z^{+}}=\frac{1}{P r^{1 / 2} R a^{1 / 2}}\left[\frac{1}{r^{+}} \frac{\partial}{\partial r^{+}}\left(r^{+} \frac{\partial \Theta}{\partial r^{+}}\right)+\frac{\partial^{2} \Theta}{\partial z^{+} \partial z^{+}}\right]$.

In Eq. (17), $\tau_{i j}{ }^{+}$is the non-dimensional stress tensor which is given by:

$\tau_{i j}{ }^{+}=\tau_{i j} L / \mu \sqrt{g \beta \Delta T_{\text {ref }} L}$,

where $r$ is the radial coordinate, $z$ axis is the vertical direction, and the axisymmetric flow is independent of the azimuthal direction $\phi$. The viscous stresses (i.e. $\tau_{r r}, \tau_{\phi \phi}, \tau_{r z}$ and $\tau_{z z}$ ) are expressed according to Eq. (5).

Figure 1 shows the schematic diagrams of the configurations for both CWT and CWHF boundary conditions. Accordingly, the governing Eqs. (16-19) are solved in a coupled manner where the two horizontal walls are adiabatic in nature (i.e. $\partial \Theta / \partial z^{+}=0$ at $z^{+}=0.0$ and $z^{+}=A R$ ), and both velocity components (i.e. $u^{+}$and $w^{+}$) are identically zero on each boundary because of the no-slip condition and impenetrability of rigid walls. For the CWHF boundary condition, the heat fluxes for vertical hot and cold walls are specified (i.e. $-\partial \Theta / \partial r^{+}=1$ and $-\partial \Theta / \partial r^{+}=1 /\left(1+L / r_{i}\right)$ at $r^{+}=r_{i} / L$ and $r^{+}=$ $r_{i} / L+1$ respectively). By contrast, the temperatures of vertical walls are specified (i.e. $\Theta=1$ and $\Theta=$ 0 at $r^{+}=r_{i} / L$ and $r^{+}=r_{i} / L+1$ respectively) for the CWT boundary condition.

\section{NUMERICAL IMPLEMENTATION}


The conservation equations (i.e. Eqs. (16-19) are solved in the framework of finite-volume methodology by using a commercial package ANSYS-FLUENT. This commercial package was previously used successfully for simulating non-Newtonian fluid flows (Hassan et al., 2013; Turan et al., 2010; Yigit et al., 2017; Yigit et al., 2015a). The convective terms are discretised using a second-order up-wind scheme, whereas the diffusive terms are discretised by a second-order central differencing scheme. The coupling of the pressure and velocity is accounted for by the well-known SIMPLE (Semi-Implicit Method for Pressure-Linked Equations) algorithm (Patankar, 1980). The convergence criteria were taken to be $10^{-6}$ for all the relative (scaled) residuals. The numerical scheme has been validated in the past with respect to the benchmark data of (de Vahl Davis, 1983) for natural convection of Newtonian fluids in square enclosures with differentially heated vertical walls and an excellent agreement (maximum difference in mean Nusselt number is found to be less than 1\%) was obtained (see Table 3 of (Turan et al., 2010). Moreover, the variation of the mean Nusselt number for Bingham fluids in square enclosures with differentially heated vertical walls subjected to the CWT boundary condition has also been compared to the corresponding results presented by (Vola et al., 2003) in a previous analysis (Turan et al., 2010) and a good agreement was reported (maximum difference in mean Nusselt number is found to be less than $3 \%$ ).

The grid independence of the results has been established based on a careful analysis of three different meshes for each $A R$ values (i.e. M1, M2 and M3) for both Newtonian and Bingham fluids. The details of the non-uniform Cartesian meshes, which have been used in current analysis, are listed in Table 2. The maximum numerical uncertainty associated with the mean Nusselt number $\overline{N u}_{i}$ based on the inner periphery for both Newtonian (i.e. $B n=0$ ) and Bingham fluids (i.e. $B n=0.2$ ) has been found to be smaller than $1 \%$ between M1, M2 and M3 mesh configurations for the ranges of parameters (i.e. $0.125 \leq r_{i} / L \leq 16, \quad 0.125 \leq A R \leq 8 \quad$ and $\quad 10^{3} \leq R a \leq 10^{6}$ at $\operatorname{Pr}=10^{3}$ ) considered here. Accordingly, the M2 mesh configuration has been used for each $A R$ for the sake of accuracy of numerical results and computational economy. Interested readers are referred to Lewis et al. (1996), Lewis et al. (2004) and Nithiarasu et al. (2016) for further information on the necessity of grid independence in numerical heat transfer problems and the methodology adopted to establish this.

\section{SCALING ANALYSIS}

A detailed scaling analysis is utilised to express the effects of $\operatorname{Ra}, \operatorname{Pr}, A R, B n, r_{i} / L$ on the mean Nusselt number $\overline{N u}_{i}$ in the current analysis. The velocity component in the vertical direction (i.e. $w$ ) can be scaled by equating the order of magnitudes of inertial and buoyancy terms as: $w \sim \sqrt{g \beta\left(T_{H}-T_{C}\right) H}$ $\left(w \sim \sqrt{g \beta q_{i} \delta_{t h} H / k}\right)$ for CWT (CWHF) boundary conditions where $\delta_{t h}$ is the thermal boundary layer thickness on the vertical walls. Based on the continuity equation one obtains: 
$\frac{1}{r} \frac{\partial(r u)}{\partial r} \sim\left(\frac{u}{r}+\frac{u}{L}\right) \sim \frac{\partial w}{\partial z} \sim \frac{w}{H}$

which leads to:

$u \sim \frac{w r}{A R(r+L)} \sim \frac{w}{A R\left(1+L / r_{i}\right)} \sim \frac{\alpha}{L} \frac{1}{\sqrt{A R}} \frac{\sqrt{R a_{C W T} P r}}{\left(1+L / r_{i}\right)} \quad$ for CWT;

$u \sim \frac{w r}{A R(r+L)} \sim \frac{w}{A R\left(1+L / r_{i}\right)} \sim \frac{\alpha}{L} \frac{1}{\sqrt{A R}} \frac{\sqrt{R a_{C W H F} P r}}{\left(1+L / r_{i}\right)} \sqrt{\frac{\delta_{t h}}{L}}$ for CWHF.

Similarly, equating the order of magnitudes of inertial and viscous terms in the vertical direction yields:

$\rho \frac{w^{2}}{H} \sim \frac{1}{\delta}\left(\tau_{y}+\mu \frac{w}{\delta}\right)$

Using Eqs. (21i) and (21ii) in Eq. (22) leads to:

$\frac{\delta_{t h}}{L} \sim \frac{1}{f_{2}} \sqrt{\frac{P r}{R a_{C W T}}}\left\{\frac{B n_{C W T}}{2}+\sqrt{\left(\frac{B n_{C W T}}{2}\right)^{2}+\sqrt{\frac{R a_{C W T} A R}{P r}}}\right\}$ for CWT;

$\left(\frac{\delta_{t h}}{L}\right)^{5 / 2} \sqrt{\frac{R a_{C W H F}}{P r}} \sim \frac{\sqrt{A R}}{f_{3}^{2}}+\frac{B n_{C W H F}}{f_{3}}\left(\frac{\delta_{t h}}{L}\right)^{1 / 2}$ for CWHF,

where $\delta$ is the hydrodynamic boundary layer thickness on the vertical wall and $f_{2}, f_{3}\left(R a, \operatorname{Pr}, B n, A R, r_{i} / L\right)$ depicts the ratio of hydrodynamic and thermal boundary thicknesses (i.e. $\left.\delta / \delta_{t h}\right)$ for CWT and CWHF configurations, respectively. Eq. (22i) clearly shows that $\delta_{t h} / L$ increases with increasing $A R\left(B n_{C W T}\right)$ for a given set values $R a$ and $P r$. Moreover, Eq. (22ii) indicates that an exact analytical solution does not exist for the CWHF configuration. However, some behaviours can be obtained based on limiting assumptions. For instances, for small values of $B n_{C W H F}$ the contribution of $B n_{C W H F}\left(\delta_{t h} / L\right)^{1 / 2} / f_{3}$ can be ignored in comparison to $\sqrt{A R} / f_{3}{ }^{2}$, which leads to $\delta_{t h} / L \sim(A R)^{0.2}(P r /$ $\left.R a_{C W H F}\right)^{0.2} f_{3}{ }^{-0.8}$. This limiting assumption also suggests that $\delta_{t h} / L$ increases with increasing $A R$ $\left(B n_{C W H F}\right)$ for a given set values $R a$ and $P r$. Interested readers refer to (Turan et al., 2014) for further information. Since the qualitative trend is expected to be the same for both CWT and CWHF configurations, the scaling analysis is continued here for cylindrical annular enclosures in the CWT configuration.

Based on the scaling estimates in Eqs. (21i) and (22i), it is possible to estimate the effective viscosity in vertical boundary layer (i.e. $\left.\mu_{e f f}{ }^{V}\right)$ in the following manner $\left(\mu_{e f f}{ }^{V} \sim \mu+\tau_{y} \delta / w\right)$ :

For CWT configuration:

$\frac{\mu_{e f f} V}{\mu} \sim\left[1+\frac{B n_{C W T}}{\sqrt{A R}} \sqrt{\frac{P r}{R a_{C W T}}}\left\{\frac{B n_{C W T}}{2}+\sqrt{\left(\frac{B n_{C W T}}{2}\right)^{2}+\sqrt{\frac{R a_{C W T} A R}{P r}}}\right\}\right.$. 
Similarly, equating order of magnitudes of inertial and viscous terms in the radial direction (i.e. $\rho u^{2} / L \sim 1 / \delta_{1}\left(\tau_{y}+\mu u / \delta_{1}\right)$ to estimate the effective viscosity in horizontal boundary layer (i.e. $\mu_{e f f}{ }^{H}$ ) in the following manner $\left(\mu_{e f f}{ }^{H} \sim \mu+\tau_{y} \delta_{1} / u\right)$ :

$\frac{\mu_{e f f^{H}}}{\mu} \sim\left[1+B n_{C W T} \sqrt{A R}\left(1+L / r_{i}\right) \sqrt{\frac{P r}{R a_{C W T}}}\left\{\frac{B n_{C W T} A R\left(1+L / r_{i}\right)^{2}}{2}+\sqrt{\left[\frac{B n_{C W T} A R\left(1+L / r_{i}\right)^{2}}{2}\right]^{2}+\sqrt{\frac{R a_{C W T} A R}{P r}}\left(1+L / r_{i}\right)^{2}}\right\}\right]$,

where $\delta_{1}$ is the hydrodynamic boundary layer thickness on the horizontal wall. Using Eq. (23), the effective Rayleigh numbers (i.e. $R a_{e f f}{ }^{V}$ and $R a_{e f f}{ }^{H}$ ) in both vertical and horizontal boundary layers can be estimated in the following manner (i.e. $R a_{\text {eff }}=\rho g \beta\left(T_{H}-T_{C}\right) L^{3} / \mu_{e f f} \alpha$ ):

$$
\begin{aligned}
& R a_{e f f}{ }^{V} \sim R a_{C W T} /\left[1+\frac{B n_{C W T}}{\sqrt{A R}} \sqrt{\frac{P r}{R a_{C W T}}}\left\{\frac{B n_{C W T}}{2}+\sqrt{\left(\frac{B n_{C W T}}{2}\right)^{2}+\sqrt{\frac{R a_{C W T} A R}{P r}}}\right\} ;\right. \\
& R a_{e f f}{ }^{H} \sim R a_{C W T} /\left[1+B n_{C W T} \sqrt{A R}\left(1+L / r_{i}\right) \sqrt{\frac{P r}{R a_{C W T}}}\left\{\frac{B n_{C W T A R\left(1+L / r_{i}\right)^{2}}}{2}+\sqrt{\left[\frac{B n_{C W T A R}\left(1+L / r_{i}\right)^{2}}{2}\right]^{2}+\sqrt{\frac{R a_{C W T R}}{P r}}\left(1+L / r_{i}\right)^{2}}\right\}\right] .
\end{aligned}
$$

Eq. (24) indicates that the effective Rayleigh number $R a_{\text {eff }}$ remains smaller than the nominal Rayleigh number and $R a_{\text {eff }}$ decreases with increasing Bn. Furthermore, Eq. (23) indicates that $\mu_{e f f}{ }^{H}$ is expected to be greater than $\mu_{e f f}{ }^{V}$ in cylindrical enclosures. This further implies that $R a_{e f f}{ }^{H}$ is expected to be smaller than $R a_{e f f} V$ in cylindrical enclosures as shown in Eq. (24).

Finally, the wall heat flux for the internal radius $q_{i}$ can be scaled as: $q_{i}=h_{i} \Delta T \sim k \Delta T / \delta_{t h}$ where $\delta_{t h}$ is the thermal boundary layer on the vertical wall. Accordingly, the scaling estimate of the mean Nusselt number can be expressed as $\overline{N u}_{i} \sim h_{i} L / k \sim L / \delta_{t h}$. It is not possible to obtain an analytical relation for $\delta_{t h}$ from Eq. (22ii) for the CWHF boundary condition but it is possible to obtain a scaling estimate of $\overline{N u}_{i}$ using Eq. (22i) for the CWT boundary condition in the following manner:

$$
\overline{N u}_{i} \sim \operatorname{Max}\left[\left(\overline{N u}_{i}\right)_{c o n d}, \frac{\left(R a_{C W T} / P r\right)^{\frac{1}{2}}}{\left[\frac{B n_{C W T}}{2}+\frac{1}{2} \sqrt{B n_{C W T}{ }^{2}+4\left(\frac{R a_{C W T} A R}{P r}\right)^{\frac{1}{2}}}\right.} f_{2}\left(R a_{C W T}, \operatorname{Pr}, B n_{C W T}, A R, r_{i} / L\right)\right] .
$$

It is worth noting that $\overline{N u}_{i}$ scaling estimation given in Eq. (25) is valid in the thermal boundary layer regime where the high values of temperature gradient are confined to two thin boundary layers adjacent to the vertical walls (Bejan and Tien, 1978). However, for shallow enclosures (i.e. $A R \ll 1$ ) the limiting condition given by $R a_{C W T} A R^{3} \rightarrow 0\left(R a_{C W H F} A R^{3} \rightarrow 0\right)$ is referred to as the parallel-flow regime (Bejan and Tien, 1978). In the parallel flow regime, vertical velocity component at the core of the enclosure 
disappears and the fluid flow in the enclosure compromise two counter flowing horizontal streams. Interested readers are referred to (Bejan and Tien, 1978; Turan et al., 2011a, 2014) for further discussion on this scaling analysis.

\section{RESULTS \& DISCUSSION}

\subsection{Effects of varying nominal Rayleigh number $R a$}

The variations of non-dimensional temperature $\theta=\left(T-T_{c e n}\right) / \Delta T_{r e f}$ and non-dimensional axial (radial) $W=w L / \alpha(U=u L / \alpha)$ velocity components along the horizontal (vertical) mid-plane for different $R a$ values at $r_{i} / L=1, A R=0.5$ and $P r=500$ are shown in Fig. 2 for Newtonian (i.e. $B n=$ $0)$ and a representative Bingham fluid case (i.e. $B n=0.05)$ for both CWT and CWHF configurations. The distributions of $\theta$ for the pure-conduction solution (where fluid flow does not affect thermal transport) are also shown in Fig. 2. Figure 2 shows that the non-dimensional temperature $\theta$ distribution between the hot and cold walls of the enclosure deviates significantly from the pure conduction solution with increasing $R a$ for both Newtonian (i.e. $B n=0$ ) and Bingham fluid (i.e. $B n=0.05)^{*}$ cases, which is indicative of the strengthening of convective thermal transport. This behaviour can also be seen from the increases in the magnitudes of $W$ and $U$ with increasing $R a$, as shown in Fig. 2. It is also worth noting that the magnitudes of $W$ and $U$ for the Bingham fluid case (i.e. $B n=0.05$ ) remain smaller than the values obtained for the Newtonian $(B n=0)$ fluid case for the same set of values of $R a, P r, A R$ and $r_{i} / L$. The viscous resistance strengthens with increasing $B n$, which is reflected in the smaller magnitudes of $W$ and $U$ in the Bingham fluid case than in the corresponding Newtonian fluid case. Furthermore, the magnitudes of $\theta, W$ and $U$ are found to be smaller in the CWHF configuration than the corresponding values in the CWT configuration, which is an indication of stronger convective thermal transport in the CWT configuration than in the CWHF configuration. This can also be confirmed by the scaling the wall heat flux on the inner periphery as $q_{i} \sim k \Delta T / \delta_{t h}$ where $\Delta T$ and $\delta_{t h}$ are the characteristic temperature difference and the thickness of thermal boundary layer on vertical walls, respectively. This implies that $\theta$ in the case of CWHF boundary condition scales as $\theta \sim \Delta T k / q_{i} L \sim q_{i} \delta_{t h} k / q_{i} L k \sim O\left(\delta_{t h} / L\right)$, whereas $\theta \sim O(1)$ for the CWT boundary condition. Accordingly, the magnitude of $\theta$ for the CWHF boundary condition is expected to decrease with increasing $R a$ due to the strengthening of buoyancy force. This smaller temperature difference between the vertical walls in the CWHF configuration induces a weaker convective transport than in the CWT configuration. Additionally, the contours of non-dimensional temperature $\theta$ and non-dimensional stream function $\Psi=\psi / \alpha$ are shown in Fig. 3 for different values of $R a$ at $r_{i} / L=1, A R=0.5$ and $\operatorname{Pr}=$ 500 for Newtonian (i.e. $B n=0$ ) and Bingham fluid case (i.e. $B n=0.05$ ) for the CWT boundary

\footnotetext{
* The lines for $R a=10^{3}$ and $R a=10^{4}$ collapse with each other and also with the pure conduction solution for $B n=0.05$ in Fig.2, since heat transfer takes place due to pure conduction for both $R a=10^{3}$ and $R a=10^{4}$ (see isotherms are parallel to the side walls in Figure 3 for $B n=0.05$ at $R a=10^{4}$ )
} 
condition. Figure 3 shows that the magnitude of $\Psi$ increases and isotherms become increasingly curved with increasing $R a$ due to the strengthening of convective thermal transport. Similar qualitative behaviour has been also observed for the CWHF boundary condition but it is not shown here for the sake of conciseness. The grey regions on the streamline plot in Fig. 3 shows the regions where $|\tau| \leq \tau_{y}$ (Mitsoulis and Zisis, 2001). The distribution of AURs is dependent on the prediction of $|\tau| \leq \tau_{y}$ which is a function of the choice of $\mu_{y i e l d} / \mu$. Therefore the grey regions should not be treated as exact unyielded zones and for this very reason Mitsoulis and Zisis (2001) termed them as the Apparently Unyielded Regions (AURs). The value of $\mu_{\text {yield }}$ affects only the size and distributions of AURs but both qualitative and quantitative distributions of stream function and isotherms remain independent of the value of $\mu_{\text {yield }} / \mu$ (at least for $\mu_{\text {yield }} / \mu \geq 10^{3}$ ). Therefore, the precise shape and size of AURs do not influence $\overline{N u}_{i}$ in the present study.

\subsection{Effects of varying Bingham number $B n$}

The variations of $\theta, W(U)$ in the radial (axial) direction at $z / L=0.5\left(\left(r-r_{i}\right) / L=0.5\right)$ are shown in Fig. 4 for different values of $B n$ and $A R$ at $r_{i} / L=1, R a=10^{6}$ and $\operatorname{Pr}=500$ for both CWT and CWHF boundary conditions. The distributions of $\theta$ for pure conduction are shown in Fig. 4 . It can be seen from Fig. 4 that $\theta$ approaches pure conduction solution with increasing $B n$, regardless of the boundary condition due to the weakening of buoyancy force relative to the viscous resistance. This is reflected in the decreasing magnitudes of $W$ and $U$ with increasing $B n$ as shown in Fig. 4 for both CWT and CWHF configurations. The contours of non-dimensional temperature $\theta$ and non-dimensional stream function $\Psi$ are shown in Fig. 5 for different values of $B n$ at $A R=0.5, r_{i} / L=1, R a=10^{6}$ and $\mathrm{Pr}=500$ for the CWT boundary condition. Figure 5 shows that the magnitude of $\Psi$ decreases and isotherms tend to be parallel to the active walls (i.e. hot and cold) due to the weakening of convective thermal transport with increasing $B n$. Accordingly, the size of AURs increases with increasing $B n$ and heat transfer takes place purely due to conduction for large values of $B n$ because flow practically stops under such a condition. These findings are also consistent with previous analyses (Yigit and Chakraborty, 2017b; Yigit et al., 2017) which dealt with natural convection of Bingham fluids in annular spaces with a square cross-section (i.e. same configuration for $A R=1.0$ ).

\subsection{Effects of varying aspect ratio $A R$}

The variations of non-dimensional temperature $\theta$ and non-dimensional axial (radial) $W(U)$ velocity component along the horizontal (vertical) mid-plane for different $A R$ values at $r_{i} / L=1, R a=10^{6}$, $B n=0.2$ and $\operatorname{Pr}=500$ are shown in Fig. 6 for both CWT and CWHF configurations. The nondimensional temperature $\theta$ distributions obtained from a pure-conduction solution (where fluid flow does not affect thermal transport) are also shown in Fig. 6 for CWT (CWHF) configurations. Figure 6 shows that the distribution of $\theta$ deviates from the pure conduction solution with increasing $A R$, which 
indicates a strengthening of convection with increasing $A R$ for both CWT and CWHF configurations. Additionally, Fig. 6 shows that the magnitudes of $\theta, W$ and $U$ in the CWHF configuration are smaller than the corresponding values obtained for the CWT configuration. This is also a confirmation of stronger convection for the CWT boundary condition than in the CWHF configuration for different $A R$ values for a given set of values of $R a, P r, B n, r_{i} / L$. Furthermore, it can be seen from Fig.6 that the magnitudes of $W$ and $U$ increase with increasing $A R$ for a given set of values of $R a, P r, B n, r_{i} / L$ for both CWT and CWHF configurations.

The variations of the mean Nusselt number based on the inner periphery $\bar{N} \bar{u}_{i}$ normalised by the corresponding value for pure conductive transport (i.e. $\left.\overline{N u}_{i} /\left(\overline{N u}_{i}\right)_{c o n d}\right)$ with $A R$ for different values $R a$ at $r_{i} / L=1$ and $\operatorname{Pr}=500$ are shown in Fig. 7 a for Newtonian fluids (i.e. $B n=0$ ) for both CWT and CWHF configurations. Figure 7a shows that a non-monotonic variation of $\overline{N u}_{i} /\left(\overline{N u}_{i}\right)_{\text {cond }}$ with $A R$ for Newtonian fluids (i.e. $B n=0$ ) in the case of CWT boundary condition, whereas a monotonic increase in $\overline{N u}_{i} /\left(\overline{N u}_{i}\right)_{\text {cond }}$ was obtained for increasing values of $A R$ in the CWHF configuration. Furthermore, the variations of $\overline{N u}_{i} /\left(\overline{N u}_{i}\right)_{\text {cond }}$ with $B n$ for different values $A R$ at $r_{i} / L=1, R a=10^{6}$ and $\operatorname{Pr}=500$ are shown in Fig. 7b for both CWT and CWHF configurations. It is worth noting that Nusselt number for pure conductive transport $\left(\overline{N u}_{i}\right)_{\text {cond }}$ also depends on $r_{i} / L$ as shown in Eq. (14) (i.e. $\left.\left(\overline{N u}_{i}\right)_{\text {cond }}=\left(L / r_{i}\right) / \ln \left(1+L / r_{i}\right)\right)$. Figure $7 \mathrm{~b}$ demonstrates that $\overline{N u}_{i} /\left(\overline{N u}_{i}\right)_{\text {cond }}$ decreases with increasing $B n$ and attains a value of unity (purely conductive thermal transport) for either equal to or greater than a threshold value of Bingham number $B n$ which is termed as $B n_{\max }$ in the current analysis. Here, $B n_{\max }$ is considered to be the value of Bingham number where $\overline{N u}_{i} /\left(\overline{N u}_{i}\right)_{\text {cond }}=1.01$ and $\overline{N u}_{i} /\left(\overline{N u}_{i}\right)_{\text {cond }}<1.01$ for $>B n_{\max }$.

It is worth noting that $B n_{\max }$ does not provide the estimation of the critical Bingham number $B n_{c}$ at which the flow ceases to take place in the context of a bi-viscosity regularisation. This is due to the fact that the flow always remains present for bi-viscosity regularisation but it no longer affects thermal advection for large values of $B n$ (Turan et al., 2011a). Thus, $B n_{\max }$ is physically different from the critical Bingham number $B n_{c}$ at which the buoyancy force becomes just sufficient to overcome the yield stress. Under this situation the fluid is unyielded throughout the domain and $\overline{N u}_{i}$ becomes equal to $\left(\overline{N u}_{i}\right)_{\text {cond }}$. For $B n=B n_{c}$, the equilibrium of buoyancy and yield stress contributions (i.e. $\rho g \beta \Delta T \sim \tau_{y} / \delta \sim \tau_{y} / L f_{2}\left(B n_{c}, P r\right)$ ) gives rise to $B n_{c} \sim \sqrt{R a / P r} f_{2}\left(B n_{c}, P r\right)$. This is consistent with the recent analytical results of (Karimfazli et al., 2015) and (Vikhansky, 2010) for the onset of natural convection of Bingham fluids in rectangular enclosures with differentially heated vertical sidewalls. However, the quantity $\left(B n_{\max }\right)_{i}$ can be scaled for the CWT boundary condition by considering $\overline{N u}_{i} \sim L / \delta_{t h} \sim L f_{2} / \delta \sim\left(\overline{N u}_{i}\right)_{\text {cond }}$. This along with Eq. (25) leads to: $\left(B n_{\text {max }}\right)_{i} \sim f_{2}\left(\overline{N u}_{i}\right)_{\text {cond }}^{-1} \sqrt{R a / P r}-\left[\sqrt{A R}\left(\overline{N u}_{i}\right)_{\text {cond }} / f_{2}\right]$ for the CWT boundary condition. It is worth 
noting that $B n>B n_{c}$ ensures that $\overline{N u}_{i} /\left(\overline{N u}_{i}\right)_{c o n d}$ remains equal to unity but it does not imply that $\overline{N u}_{i} /\left(\overline{N u}_{i}\right)_{\text {cond }}>1$ when $B n$ is smaller than $B n_{c}$. In the current analysis, $\overline{N u}_{i} /\left(\overline{N u}_{i}\right)_{\text {cond }}$ is only attains values greater than unity when $B n$ is smaller than $\left(B n_{\max }\right)_{i}$, and fluid may remain in a yielded state under the condition $\left(B n_{\max }\right)_{i}<B n<B n_{c}$ even though the flow is weak enough to result in a unity value of $\overline{N u}_{i} /\left(\overline{N u}_{i}\right)_{\text {cond }}$. Interested readers are referred to (Turan et al., 2011a, 2014; Yigit and Chakraborty, 2017b) for further information.

Moreover, as shown in Figs. 6 and 7, the variation of $\bar{N} \bar{u}_{i} /\left(\overline{N u}_{i}\right)_{\text {cond }}$ shows a non-monotonic trend with $A R$ for a given set of values of $R a, P r, B n, r_{i} / L$ for both Newtonian (i.e. $B n=0$ ) and Bingham fluids in the case of CWT boundary condition, whereas a monotonic increase in $\overline{N u}_{i} /\left(\overline{N u}_{i}\right)_{\text {cond }}$ was obtained for increasing values of $A R$ for the CWHF configuration. For example, the highest value of $\overline{N u}_{i} /\left(\overline{N u}_{i}\right)_{\text {cond }}$ is obtained at $A R=2$ for $r_{i} / L=1, R a=10^{6}, B n=0.2$ and $\operatorname{Pr}=500$ in the CWT configuration. By contrast, the highest value of $\bar{N} \bar{u}_{i} /\left(\bar{N}_{i}\right)_{\text {cond }}$ is obtained for the highest $A R$ case in the CWHF configuration, as indicated by an asterisk in Fig. 6. This is consistent with previous analyses on the influences of aspect ratio and boundary condition on natural convection in rectangular enclosures for both Newtonian (i.e.Bn =0) and Bingham fluids (Turan et al., 2011a, 2014). The non-monotonic (monotonic) $A R$ dependence of $\bar{N}_{i} /\left(\bar{N}_{i}\right)_{\text {cond }}$ for the CWT (CWHF) boundary condition can be explained in the following manner using the energy flux integral. The energy flux integral at the horizontal mid-plane can be given as:

$\dot{Q}=\dot{Q}_{c o n v}+\dot{Q}_{c o n d}=\int_{r_{i}}^{r_{o}} \rho c_{p} T w 2 \pi r d r-\int_{r_{i}}^{r_{o}} k\left(\frac{\partial T}{\partial z}\right) 2 \pi r d r$,

where the first term on the right hand side represents the effects of convective transport, whereas the second term on the right hand side accounts for thermal conduction. Here, $\dot{Q}_{c o n v}$ and $\dot{Q}_{c o n d}$ can be scaled in the following manner:

$$
\begin{aligned}
& \dot{Q}_{c o n v, C W T} \sim \pi\left(2 r_{i}+L\right) \rho c_{p} \Delta T w \delta \sim k \Delta T \pi\left(2 r_{i}+L\right) \sqrt{A R} P r\left[\frac{B n_{C W T}}{2}+\sqrt{\left(\frac{B n_{C W T}}{2}\right)^{2}+\sqrt{\frac{R a_{C W T} A R}{P r}}}\right] \\
& \dot{Q}_{c o n v, C W H F} \sim \pi\left(2 r_{i}+L\right) \frac{\rho c_{p} w q_{i} \delta_{t h} \delta}{k} \sim q_{i} L \pi\left(2 r_{i}+L\right) f_{3} \sqrt{R a_{C W H F} \operatorname{PrAR}}\left(\delta_{t h} / L\right)^{5 / 2} ; \\
& \dot{Q}_{\text {cond }, C W T} \sim k \Delta T \pi\left(2 r_{i}+L\right) / A R ; \\
& \dot{Q}_{\text {cond }, C W H F} \sim \pi\left(2 r_{i}+L\right) \frac{q_{i} \delta_{t h}}{H} \sim q_{i} L \pi\left(2 r_{i}+L\right)\left(\delta_{t h} / L\right)(1 / A R) .
\end{aligned}
$$

The above scaling estimates indicate that the convective transport strengthens with increasing $A R$, whereas thermal diffusion weakens with increasing $A R$ in the case of CWT boundary condition. The highest value of $\overline{N u}_{i} /\left(\overline{N u}_{i}\right)_{\text {cond }}$ takes place for an optimum value of aspect ratio (i.e. $\left.A R_{\max }\right)$ due to the competition between an enhancement in advection and a weakening of thermal diffusion with an increase in $A R$. By contrast, the strengthening (weakening) of $\dot{Q}_{c o n v, C W H F}\left(\dot{Q}_{c o n d, C W H F}\right)$ with increasing $A R$ in the 
CWHF configuration is stronger (weaker) than $\dot{Q}_{c o n v, C W T}\left(\dot{Q}_{c o n d, C W T}\right)$ in the CWT configuration. For example, in Newtonian fluids (i.e. $B n=0), \dot{Q}_{c o n v}$ scales as $\dot{Q}_{c o n v, C W T} \sim k \Delta T \pi\left(2 r_{i}+\right.$ L) $R a_{C W T}{ }^{0.25} \operatorname{Pr}^{0.75} A R^{0.75}$ and $\dot{Q}_{c o n v, C W H F} \sim q_{i} L \pi\left(2 r_{i}+L\right) \operatorname{PrARf}_{3}{ }^{-1}$ for CWT and CWHF boundary conditions respectively. Similarly, $\dot{Q}_{c o n d}$ scales as $\dot{Q}_{c o n d, C W T} \sim k \Delta T \pi\left(2 r_{i}+L\right) A R^{-1}$ and $\dot{Q}_{\text {cond }, C W H F} \sim q_{i} L \pi\left(2 r_{i}+L\right) \operatorname{Pr}^{0.2} R a^{-0.2} A R^{-0.8} f_{3}^{-0.8}$ for CWT and CWHF boundary conditions respectively. The quantities $k \Delta T$ and $q_{i} L$ are kept unaltered for CWT and CWHF configurations, and thus, the strengthening (weakening) of the convective (conductive) transport with increasing $A R$ in the CWHF configuration is stronger (weaker) than that in the CWT configuration for a given set of values of $R a$ and $P r$ for Newtonian fluids. A qualitatively similar behaviour has also been observed here for Bingham fluids. Thus, $\bar{N}_{i} /\left(\bar{N} \bar{u}_{i}\right)_{\text {cond }}$ increases monotonically with increasing $A R$ in the CWHF configuration. This aspect is also shown in Fig. 8 with the contours of non-dimensional stream function $\Psi$ with AURs for different $A R$ values at $r_{i} / L=1, R a=10^{6}, B n=0.2$ and $P r=500$ for the CWHF boundary condition. Findings of Figs 6-8 are in qualitative agreement with previous analyses (Turan et al., 2011a, 2014) on rectangular enclosures for both Newtonian (i.e. $B n=0$ ) and Bingham fluids.

\subsection{Effects of varying $r_{i} / L$}

The variations of non-dimensional temperature $\theta$ along the horizontal mid-plane for different $r_{i} / L$ values are shown in Fig. 9 for $A R=0.5, R a=10^{6}, B n=0.1$ and $P r=500$ in the case of both CWT and CWHF configurations. Figure 9 indicates that the temperature difference increases with increasing $r_{i} / L$ for the Bingham fluid case (i.e. $B n=0.1$ ) in the CWHF configuration. It is important to understand that $r_{i} / L$ dependence of the temperature difference between vertical walls $\Delta T$ due to pure conductive transport in order to explain the wall curvature effects on the temperature difference between the vertical walls. It is worth noting that the temperature difference between the vertical walls $\Delta T$ is dependent on the internal radius of the cylindrical annular enclosure when the thermal transport occurs purely by thermal conduction. The thermal resistance due to conduction in axisymmetric cylindrical geometry is given by $\ln \left(r_{o} / r_{i}\right) / 2 \pi k L$, which suggests that the steady state heat flow rate for pure conductive transport is given by: $\dot{Q}=\Delta T_{\text {cond }} /\left\{\ln \left(r_{o} / r_{i}\right) / 2 \pi k L\right\}$. Furthermore, $\dot{Q}$ can be expressed in terms of heat flux on the inner periphery as: $\dot{Q}=q_{i} 2 \pi r_{i} L$, which leads to:

$$
\Delta T_{\text {cond }}=\frac{q_{i} L\left(r_{i} / L\right)}{k} \ln \left(1+\frac{L}{r_{i}}\right) \text {. }
$$

Eq. (28) indicates that $\Delta T_{\text {cond }}$ increases with decreasing $r_{i} / L$. As the heat transfer coefficient $h_{i}=$ $q_{i} / \Delta T$ for the convective transport is expected to be greater than that for the pure conductive transport $\left(h_{i}\right)_{\text {cond }}=q_{i} / \Delta T_{\text {cond }}$, the temperature difference between the vertical walls remains smaller than that in the case of purely conductive transport in the boundary layer regime of convection. Furthermore, the variations of non-dimensional axial (radial) $W(U)$ velocity components along the horizontal (vertical) mid-plane for different $r_{i} / L$ values are shown in Fig. 9 for $A R=0.5, R a=10^{6}$ 
$B n=0.1$ and $\operatorname{Pr}=500$ in the case of both CWT and CWHF configurations. It can be seen from Fig. 8 that $U$ increases while $W$ decreases with increasing $r_{i} / L$ Bingham fluid case (i.e. $B n=0.1$ ) in both CWT and CWHF configurations. These numerical findings can be confirmed from the scaling estimation of the radial velocity component given by Eq. 21 (i.e. $u \sim w\left(1+L / r_{i}\right)$ ) regardless of the boundary condition. As the variations $W$ and $U$ with $r_{i} / L$ exhibit opposite behaviours, as shown in the Fig.9, the relative contributions of thermal transport due to convection and conduction for different values of $r_{i} / L$ can be explained from the variations of $\overline{N u}_{i} /\left(\bar{N}_{i}\right)_{\text {cond }}$ with $r_{i} / L$. It is worth noting that $\left(\overline{N u}_{i}\right)_{\text {cond }}$ increases with decreasing $r_{i} / L$ (see Eq. 14). Therefore, the variations of $\overline{N u}_{i} /\left(\overline{N u}_{i}\right)_{\text {cond }}$ with $r_{i} / L$ for different values of $B n$ at $A R=0.5, R a=10^{6}$ and $\operatorname{Pr}=500$ are shown in Fig. 10 for both CWT and CWHF configurations. Figure 10 shows that $\overline{N u}_{i} /\left(\overline{N u}_{i}\right)_{\text {cond }}$ increases with increasing $r_{i} / L$ and approaches asymptotically to the value of the mean Nusselt number $\overline{N u}_{r e c}$ for the corresponding rectangular enclosures (i.e. in the limit of $r_{i} / L \rightarrow \infty$ ). This suggests that the relative contribution of convection to the overall thermal transport increases with increasing $r_{i} / L$ for both Newtonian (i.e. $B n=$ 0 ) and Bingham fluids. This is in a full agreement with previous results for Newtonian fluids (de Vahl Davis and Thomas, 1969; Keyhani et al., 1983; Nagendra et al., 1970; Satya Sai et al., 1993) and also for Bingham fluids (Yigit and Chakraborty, 2017b; Yigit et al., 2017) at $A R=1$.

\subsection{Correlation of the mean Nusselt Number $\overline{\boldsymbol{N}} \bar{u}_{i}$}

Bejan and Tien (1978) proposed the following correlation for the mean Nusselt number $\overline{N u}_{R e c}$ on natural convection of Newtonian (i.e. $B n=0$ ) fluids in rectangular enclosures with differentially heated vertical walls for the CWT boundary condition:

$\overline{N u}_{R e c}=1+\left[\left(\frac{R a_{C W T^{2} A R^{8}}}{362880}\right)^{n}+\left(0.623 R a_{C W T}{ }^{1 / 5} A R^{-2 / 5}\right)^{n}\right]^{1 / n}$ where $n=-0.386$.

It is shown in elsewhere (Fig. 10 of (Turan et al., 2014) that Eq. (29) captures the variation of $\overline{N u}_{R e c}$ with $A R$ satisfactorily for $A R \leq 1$. It is worth noting that Eq. (29) underpredicts the value of $\overline{N u}_{R e c}$ for the aspect ratios of the order of unity (i.e. $A R \sim 1$ ). Also, one of the most used $\overline{N u}_{R e c}$ correlations for tall (i.e. $A R \gg 1$ ) enclosures was proposed by Elsherbiny et al. (1982) in the following manner:

$\overline{N u}_{R e c}=\operatorname{Max}\left(N u_{1 c}, N u_{2 c}, N u_{3 c}\right)$;

$N u_{1 c}=0.0605 R a_{C W T}^{1 / 3}$;

$N u_{2 c}=\left[1+\left(\frac{0.104 R a_{C W T}^{0.293}}{1+\left(6310 / R a_{C W T}\right)^{1.36}}\right)^{3}\right]^{1 / 3}$

$N u_{3 c}=0.242\left(R a_{C W T} / A R\right)^{0.272}$.

The $\overline{N u}_{R e c}$ correlation given by Eq. (30) satisfactorily captures the variation of $\overline{N u}_{R e c}$ with $A R$ for $A R \geq 2$ as it is shown in elsewhere (Fig. 10 of (Turan et al., 2014)). Similarly, Turan et al. (2014) proposed the following correlation for $\overline{N u}_{R e c}$ for natural convection of Newtonian (i.e. $B n=0$ ) fluids in rectangular enclosures with differentially heated vertical walls for the CWHF boundary condition: 
For $1 / 8 \leq A R \leq 1 ; \overline{N u}_{R e c}=N u_{A}$ for $R a_{C W H F} A R^{3}<10^{3}, \overline{N u}_{R e c}=N u_{B}$ for $R a_{C W H F} A R^{3} \geq 10^{3}$;

$N u_{A}=1+R a_{C W H F}^{2} A R^{8} / 362880$;

$N u_{B}=0.209 R a_{C W H F}^{0.249}\left(\frac{P r}{1+P r}\right)^{0.031}\left[c_{B}(1-A R)^{c_{c}}+1\right]$;

where $c_{B}=-1.168, c_{C}=0.683 R a^{0.089}{ }_{C W H F}$.

For $1<A R \leq 8$

$\overline{N u}_{R e c}=0.209 R a_{C W H F}^{0.249}\left(\frac{P r}{1+P r}\right)^{0.031}\left[c_{A} \ln A R+1\right]$,

where $c_{A}=0.737 R a_{C W H F}^{-0.189}$.

The correlation proposed in Eqs. (29-31) can be used for $r_{i} / L \gg 1$ as $\overline{N u}_{i}$ is expected to approach $\overline{N u}_{R e c}$ and $\left(\overline{N u}_{i}\right)_{\text {cond }}$ approaches unity (i.e. $\left.\lim _{r_{i} / L \rightarrow \infty}\left[\left(L / r_{i}\right) / \ln \left(1+L / r_{i}\right)\right]=1.0\right)$ in the limit of $r_{i} / L \rightarrow \infty$. In this study, the mean Nusselt number $\left(\overline{N u}_{i}\right)_{B n=0}$ for natural convection of Newtonian fluids in rectangular cross-sectional cylindrical enclosures is parameterised in the following manner:

$\left(\bar{N} \bar{u}_{i}\right)_{B n=0}=\left(\bar{N} \bar{u}_{R e c}\right)_{B n=0} \frac{\left[m_{0}\left(L / r_{i}\right)\right]^{k_{0}}}{\ln \left[1+\left(m_{0}\left(L / r_{i}\right)\right)^{k_{0}}\right]}$.

For CWT configuration;

$m_{0}=[0.712+0.016 \ln R a]^{\left(\overline{N u}_{R e c}\right)_{B n=0}-1}$ and $k_{0}=[0.656+0.022 \ln R a]^{\left(\overline{N u}_{R e c}\right)_{B n=0}-1}$.

For CWHF configuration;

$m_{0}=\left[0.151(\ln R a)^{0.66}\right]^{\left(\overline{N u}_{R e c}\right)_{B n=0}-1}$ and $k_{0}=[0.694+0.016 \ln R a]^{\left(\overline{N u}_{R e c}\right)_{B n=0}-1}$.

Equation (32) is not only valid for $0.125 \leq r_{i} / L \leq 16$ but also remains applicable for rectangular enclosures because $\overline{N u}_{i}$ will be equal to the $\overline{N u}_{R e c}$ and $\left(\overline{N u}_{i}\right)_{\text {cond }}$ will be equal to unity (i.e. $\left.\lim _{r_{i} / L \rightarrow \infty}\left[\left(L / r_{i}\right) / \ln \left(1+L / r_{i}\right)\right]=1.0\right)$ in the limit of $r_{i} / L \rightarrow \infty$. The predictions of Eq. (32) are shown in Fig. 11 for both CWT and CWHF configurations, which indicate that this correlation satisfactorily predicts $\overline{N u}_{i} /\left(\overline{N u}_{i}\right)_{\text {cond }}$ (i.e. $\left.R^{2}>0.99\right)$ for Newtonian fluids in rectangular crosssectional cylindrical annular enclosures for $10^{3} \leq R a \leq 10^{6}, 0.25 \leq A R \leq 8$ and $0.125 \leq r_{i} / L \leq 16$. It is worth noting that Eq. (32) underpredicts for $A R<1$, since the correlation given by Eq. (29) underpredicts the value of $\overline{N u}_{R e c}$ for $A R<1$ in the case of Newtonian fluids (i.e. $B n=0$ ) (see Fig. 10 of (Turan et al., 2014).

Using Eq. (25) one can obtain a scaling estimation of $\overline{N u}_{i}$ for Bingham fluids in the boundary layer regime for the CWT boundary condition in the following manner:

$\overline{N u}_{i} \sim \operatorname{Max}\left[\left(\overline{N u}_{i}\right)_{\text {cond }}, \frac{\left(\overline{N u}_{i}\right)_{B n=0}}{\left[\frac{B n^{*}}{2}+\frac{1}{2} \sqrt{B n^{* 2}+4}\right]} f_{2}\left(R a, \operatorname{Pr}, A R, B n, r_{i} / L\right)\right]$,

where $B n^{*}=B n /\left[(R a A R / P r)^{1 / 4}\right]$.

Eq. (33) is extended to correlate $\bar{N}_{i}$ for natural convection of Bingham fluids in rectangular crosssectional cylindrical annular enclosures for $0 \leq B n \leq B n_{\text {max }}$ : 
$\frac{\left[\overline{N u}_{i} /\left(\overline{N u}_{i}\right)_{\text {cond } d}\right]-1}{\left[\left(\overline{N u}_{i}\right)_{B n=0} /\left(\overline{N u}_{i}\right)_{\text {cond }}\right]-1}=\frac{2\left[1-\left(B n^{*} / B n_{\text {max }}^{*}\right)^{b_{i}}\right]^{c_{i}}}{B n^{*}+\sqrt{B n^{* 2}+4}}$ when $\left(\overline{N u}_{i}\right)_{B n=0} /\left(\overline{N u}_{i}\right)_{\text {cond }}>1$,

$\overline{N u}_{i} /\left(\overline{N u}_{i}\right)_{\text {cond }}=1$ when $\left(\overline{N u}_{i}\right)_{B n=0} /\left(\overline{N u}_{i}\right)_{\text {cond }}=1$,

where $B n_{\text {max }}^{*}=B n_{\max }(R a A R / P r)^{-1 / 4}, b_{i}$ and $c_{i}$ are the correlation parameters. Turan et al. (2014) proposed the following correlation for threshold value of Bingham number $\left(B n_{\max }^{*}\right)_{R e c}$ for rectangular enclosures, which is valid for both CWT and CWHF configurations:

$\left(B n_{\text {max }}^{*}\right)_{\text {Rec }}=[0.0 .19+0.01 \operatorname{erf}(2 A R-2)] R a^{0.31} \operatorname{Pr}^{-0.21} A R^{-0.25}$.

It is worth noting that Turan et al. (2014) used the $\overline{N u}_{R e c}=1.0$ as the criterion for $\left(B n_{\max }^{*}\right)_{\operatorname{Rec}}$ in Eq. (35) but this is different from the criterion followed here (i.e. where $\overline{N u}_{i} /\left(\overline{N u}_{i}\right)_{\text {cond }}=1.01$ ). This correlation has been modified here in the following manner based on the revised $\overline{N u}_{i} /\left(\overline{N u}_{i}\right)_{\text {cond }}$ criterion (i.e. $\left.\overline{N u}_{i} /\left(\overline{N u}_{i}\right)_{\text {cond }}=1.01\right)$.

$\left(B n_{\text {max }}^{*}\right)_{R e c}=c_{1} R a^{0.31} \mathrm{Pr}^{-0.21} A R^{-0.25}$ for CWT configuration,

$\left(B n_{\text {max }}^{*}\right)_{\text {Rec }}=c_{2} R a^{0.31} \mathrm{Pr}^{-0.21} A R^{-0.25}$ for CWHF configuration,

where $c_{1}$ and $c_{2}$ are the correlation parameters, which are listed in Table 3. The correlation proposed in Eq. (36) can be used for $r_{i} / L \gg 1$ in the limit of $r_{i} / L \rightarrow \infty$. In this study, $\left(B n_{\max }^{*}\right)_{i}$ is expressed using Eq. (36) in the following manner:

$\left(B n_{\max }\right)_{i}=\left(B n_{\max }\right)_{R e c}-m_{1}\left(L / r_{i}\right)^{k_{1}}$ for CWT configuration,

$\left(B n_{\max }\right)_{i}=\left(B n_{\max }\right)_{R e c} /\left[1+m_{2}\left(L / r_{i}\right)^{k_{2}}\right]$ for CWHF configuration,

where $m_{1}, m_{2}, k_{1}$ and $k_{2}$ are the correlation parameters, which are listed in Table 4. Equations (37) and (38) are not only valid for $0.125 \leq r_{i} / L \leq 16$ but also remain applicable to rectangular enclosures because $\left(B n_{\max }\right)_{i}$ becomes equal to the $\left(B n_{\max }\right)_{\operatorname{Rec}}\left(\right.$ i.e. $\left.\lim _{r_{i} / L \rightarrow \infty}\left[\left(L / r_{i}\right) / \ln \left(1+L / r_{i}\right)\right]=1.0\right)$ in the limit of $r_{i} / L \rightarrow \infty$. The predictions of Eqs. (37) and (38) are shown in Fig. 12 for both CWT and CWHF configurations, which indicate that this correlation satisfactorily predicts $\left(B n_{\max }\right)_{i}$ (i.e. $R^{2}>$ 0.99 ) for rectangular cross-sectional cylindrical annular enclosures for $10^{4} \leq R a \leq 10^{6}, 0.5 \leq A R \leq$ 8 and $0.125 \leq r_{i} / L \leq 16$.

Turan et al. (2014) proposed the mean Nusselt number $\overline{N u}_{R e c}$ correlation for natural convection of Bingham fluids in rectangular enclosures in the following manner by utilising Eq. (34):

For CWT configuration;

$\frac{\overline{N u}_{R e c}-1}{\left(\overline{\left.N u_{R e c}\right)_{B n=0}-1}\right.}=\left\{\left[1-\left(\frac{1}{4.55}\right)^{-2}\left(B n^{* *}\right)_{C W T}\right]^{2 / n_{1}}+\left[\frac{2\left[1-\left(B n^{*} / B n_{m a x}^{*}\right)^{b_{0}}\right]^{b_{1}}}{B n^{*}+\sqrt{B n^{* 2}+4}}\right]^{1 / n_{1}}\right\}^{n_{1}}$.

In Eq. (39), $n_{1}=-0.02$ is when $\overline{N u}_{R e c}>1$ and $\overline{N u} \overline{R e c}_{R}=1$ is when $\left(\overline{N u}_{R e c}\right)_{B n=0}=1 ; b_{0}=1.37$ and $b_{1}=0.676 R a_{C W T}^{0.131} \operatorname{Pr}^{0.11} ;\left(B n^{* *}\right)_{C W T}=B n_{C W T} /\left[\left(R a_{C W T} / P r\right)^{1 / 2} A R^{2}\right]$.

For CWHF configuration;

$\left.\frac{\overline{N u}_{R e c}-1}{\left(\overline{\left.N u_{R e c}\right)_{B n=0}-1}\right.}=\left\{\left[1-\left(\frac{1}{5.5}\right)^{-2}\left(B n^{* *}\right)_{C W H F}\right]^{2 / n_{2}}+\left[\frac{2\left[1-\left(B n^{*} / B n_{m a x}^{*}\right)^{b_{2}}\right.}{B n^{*}+\sqrt{B n^{* 2}+4}}\right]^{b_{3}}\right\}^{1 / n_{2}}\right\}^{n_{2}}$. 
In Eq. (40), $b_{2}=0.867$ and $b_{3}=0.25 R a_{C W H F}{ }^{0.207} \operatorname{Pr}^{0.062} ;\left(B n^{* *}\right)_{C W H F}=B n_{C W H F} /\left[\left(R a_{C W H F} /\right.\right.$ $\left.P r)^{1 / 2} A R^{2}\right]$, and $n_{2}=-0.02$ when $\overline{N u}_{R e c}>1$ and $\overline{N u}_{R e c}=1$ when $\left(\overline{N u}_{R e c}\right)_{B n=0}=1$.

It is worth noting that first and second terms on the right hand side of Eqs. (39) and (40) account for the contributions of the parallel flow and boundary layer regimes of convection respectively. In current study, $\overline{N u}_{R e c}$ is replaced by $\overline{N u}_{i} /\left(\overline{N u}_{i}\right)_{\text {cond }}$, and $b_{0}, b_{1}, b_{2}$ and $b_{3}$ (see Eqs. (39) and (40)) are modified in the following form for evaluating for natural convection of Bingham fluids in cylindrical enclosures:

$\left(b_{0}\right)_{i}=1.37 /\left[1+k_{3}\left(L / r_{i}\right)^{0.01}\right]$ and $\left(b_{1}\right)_{i}=\left(0.676 R a_{C W T}{ }^{0.131} \operatorname{Pr}^{0.11}\right) /\left[1+k_{4}\left(L / r_{i}\right)^{0.01}\right]$;

$\left(b_{2}\right)_{i}=0.867 /\left[1+k_{5}\left(L / r_{i}\right)^{0.01}\right]$ and $\left(b_{3}\right)_{i}=\left(0.25 R a_{C W H F}{ }^{0.207} \operatorname{Pr}^{0.062}\right) /\left[1+k_{6}\left(L / r_{i}\right)^{0.01}\right]$,

where $k_{3}, k_{4}, k_{5}$ and $k_{6}$ are the correlation parameters. The resultant correlation for $\overline{N u}_{i} /\left(\overline{N u}_{i}\right)_{\text {cond }}$ is summarised in Table 5. The correlation in Table 5 is not only valid for $0.125 \leq r_{i} / L \leq 16$ but also remains applicable for rectangular enclosures because $\left(b_{0}\right)_{i},\left(b_{1}\right)_{i},\left(b_{2}\right)_{i},\left(b_{3}\right)_{i}$ become $b_{0}, b_{1}, b_{2}, b_{3}$ respectively (i.e. $\left.\lim _{r_{i} / L \rightarrow \infty}\left[\left(L / r_{i}\right) / \ln \left(1+L / r_{i}\right)\right]=1.0\right)$ in the limit of $r_{i} / L \rightarrow \infty$. Accordingly, $\overline{N u}_{i}$ will be equal to the $\overline{N u}_{R e c}$ and $\left(\overline{N u}_{i}\right)_{c o n d}$ assumes a value of unity (i.e. $\lim _{r_{i} / L \rightarrow \infty}\left[\left(L / r_{i}\right) / \ln (1+\right.$ $\left.\left.\left.L / r_{i}\right)\right]=1.0\right)$ in the limit of $r_{i} / L \rightarrow \infty$. The predictions of $\overline{N u}_{i} /\left(\overline{N u}_{i}\right)_{c o n d}$ correlation listed in Table 5 are shown in Fig. 13 for both CWT and CWHF configurations, which indicate that this correlation satisfactorily predicts $\overline{N u}_{i} /\left(\overline{N u}_{i}\right)_{\text {cond }}$ (i.e. $R^{2}>0.98$ ) for Bingham fluids in rectangular crosssectional cylindrical annular enclosures for $10^{3} \leq R a \leq 10^{6}, 0.5 \leq A R \leq 8$ and $0.125 \leq r_{i} / L \leq 16$.

\section{CONCLUSIONS}

The effects of boundary condition (i.e. CWT and CWHF) and aspect ratio $(=H / L$ where $H$ is the height of the enclosure and $L$ is the difference between inner and outer radius) on steady-state laminar natural convection of Bingham fluids in rectangular cross-sectional cylindrical annular enclosures with differentially heated vertical side walls have been numerically investigated in this analysis. It is found that the mean Nusselt number based on the inner periphery $\overline{N u}_{i}$ increases (decreases) with increasing $R a$ $(B n)$ due to the strengthening of buoyancy force (viscous resistance), regardless of the boundary condition for the vertical walls. Accordingly, the mean Nusselt number $\overline{N u}_{i}$ normalised by the corresponding Nusselt number due to pure conductive transport (i.e. $\left.\overline{N u}_{i} /\left(\overline{N u}_{i}\right)_{\text {cond }}\right)$ decreases with increasing $B n$, and attains a value of unity (purely conductive thermal transport) for large values of $B n$ irrespective of the boundary condition. This behaviour is obtained because the fluid flow practically stops and thermal transport becomes conduction-dominated for large values of $B n$ for both CWT and CWHF configurations. Additionally, $\overline{N u}_{i} /\left(\overline{N u}_{i}\right)_{\text {cond }}$ increases with increasing $r_{i} / L$ before approaching an asymptotic value equal to the mean Nusselt number for rectangular enclosures in the limit of $r_{i} / L \rightarrow \infty$. This indicates that the ratio of convective to diffusive thermal transport increases with increasing $r_{i} / L$ for both Newtonian (i.e. $B n=0$ ) and Bingham fluids for both CWT and CWHF configurations. The effects of aspect ratio $A R$ have also been investigated in detail and it is found that $\overline{N u}_{i} /\left(\overline{N u}_{i}\right)_{\text {cond }}$ shows a 
non-monotonic trend (i.e. an increase with increasing $A R$ before reaching a maximum and subsequently shows a decreasing trend) with increasing $A R$ for a given set of values of $R a, P r, r_{i} / L$ for both Newtonian (i.e. $B n=0)$ and Bingham fluids for the CWT boundary condition, whereas $\overline{N u}_{i} /\left(\overline{N u}_{i}\right)_{\text {cond }}$ increases monotonically with increasing $A R$ in the CWHF configuration. The influences of convective thermal transport strengthen whereas thermal diffusive transport weakens with increasing $A R$ in this configuration, and these competing effects are responsible for the non-monotonic $\overline{N u}_{i} /\left(\overline{N u}_{i}\right)_{\text {cond }}$ variation with $A R$ in the CWT configuration. However, the strengthening of convective transport with increasing $A R$ dominates over the weakening of thermal diffusion in the CWHF configuration for the aspect ratio range considered here. Furthermore, conductive thermal transport plays a dominant role for small values of $A R$ in the case of both CWT and CWHF boundary conditions. Detailed scaling analysis is utilised to explain the observed influences of $R a, B n, r_{i} / L$ and $A R$ on the mean Nusselt number $\overline{N u}_{i}$ and new correlations have been proposed for the mean Nusselt number $\overline{N u}_{i}$, which have been shown to predict numerical findings accurately for the range of parameters considered here.

\section{REFERENCES}

Balmforth, N.J., Rust, A.C., (2009), "Weakly nonlinear viscoplastic convection", Journal of NonNewtonian Fluid Mechanics, Vol.158, pp.36-45.

Barnes, H.A., (1999), "The yield stress-a review or ' $\pi \alpha v \tau \alpha \rho \varepsilon \iota^{\prime}$ - everything flows?", Journal of NonNewtonian Fluid Mechanics, Vol.81, pp.133-178.

Bejan, A., Tien, C.L., (1978), "Laminar Natural Convection Heat Transfer in a Horizontal Cavity with Different End Temperatures", Journal of Heat Transfer, Vol.100, pp.641-647.

Catton, I., Ayyaswamy, P.S., Clever, R.M., (1974), "Natural convection flow in a finite, rectangular slot arbitrarily oriented with respect to the gravity vector", International Journal of Heat and Mass Transfer, Vol.17, pp.173-184.

Darbouli, M., Métivier, C., Piau, J.-M., Magnin, A., Abdelali, A., (2013), "Rayleigh-Bénard convection for viscoplastic fluids", Physics of Fluids, Vol.25, pp.023101.

de Vahl Davis, G., (1983), "Natural convection of air in a square cavity: A bench mark numerical solution", International Journal for Numerical Methods in Fluids, Vol.3, pp.249-264.

de Vahl Davis, G., Thomas, R.W., (1969), "Natural Convection between Concentric Vertical Cylinders", The Physics of Fluids, Vol.12, pp.II-198-II-207.

Elsherbiny, S.M., Raithby, G.D., Hollands, K.G.T., (1982), "Heat Transfer by Natural Convection Across Vertical and Inclined Air Layers", Journal of Heat Transfer, Vol.104, pp.96-102.

Hassan, M.A., Pathak, M., Khan, M.K., (2013), "Natural Convection of Viscoplastic Fluids in a Square Enclosure", Journal of Heat Transfer, Vol.135, pp.122501-122501-122512.

Hassan, M.A., Pathak, M., Khan, M.K., (2015), "Rayleigh-Benard convection in Herschel-Bulkley fluid", Journal of Non-Newtonian Fluid Mechanics, Vol.226, pp.32-45.

Huilgol, R.R., Kefayati, G.H.R., (2015), "Natural convection problem in a Bingham fluid using the operator-splitting method", Journal of Non-Newtonian Fluid Mechanics, Vol.220, pp.22-32.

Karimfazli, I., Frigaard, I.A., Wachs, A., (2015), "A novel heat transfer switch using the yield stress", Journal of Fluid Mechanics, Vol.783, pp.526-566.

Kebiche, Z., Castelain, C., Burghelea, T., (2014), "Experimental investigation of the Rayleigh-Bénard convection in a yield stress fluid", Journal of Non-Newtonian Fluid Mechanics, Vol.203, pp.923.

Keyhani, M., Kulacki, F.A., Christensen, R.N., (1983), "Free Convection in a Vertical Annulus With Constant Heat Flux on the Inner Wall", Journal of Heat Transfer, Vol.105, pp.454-459.

Lewis, R.W., Morgan, K., Thomas, H.R., Seetharamu, K.N., (1996), The Finite Element Method in Heat Transfer Analysis John Wiley \& Sons Inc., Chichester. 
Lewis, R.W., Nithiarasu, P., Seetharamu, K.N., (2004), Fundamentals of the Finite Element Method for Heat and Fluid Flow, John Wiley \& Sons Inc., Chichester.

Mitsoulis, E., Zisis, T., (2001), "Flow of Bingham plastics in a lid-driven square cavity", Journal of Non-Newtonian Fluid Mechanics, Vol.101, pp.173-180.

Nagendra, H.R., Tirunarayanan, M.A., Ramachandran, A., (1970), "Free convection heat transfer in vertical annuli", Chemical Engineering Science, Vol.25, pp.605-610.

Nithiarasu, P., Lewis, R.W., Seetharamu, K.N., (2016), Fundamentals of the Finite Element Method for Heat and Mass Transfer, 2nd Edition, John Wiley \& Sons Inc., Chichester.

O'Donovan, E.J., Tanner, R.I., (1984), "Numerical study of the Bingham squeeze film problem", Journal of Non-Newtonian Fluid Mechanics, Vol.15, pp.75-83.

Ostrach, S., (1988), "Natural Convection in Enclosures", Journal of Heat Transfer, Vol.110, pp.11751190.

Papanastasiou, T.C., (1987), "Flows of Materials with Yield", Journal of Rheology, Vol.31, pp.385404.

Patankar, S.V., (1980), Numerical Heat Transfer and Fluid Flow, Hemisphere, Washington D.C.

Satya Sai, B.V.K., Seetharamu, K.N., Aswatha Narayana, P.A., Reddy, J.N., (1993), "Finite element analysis of the effect of radius ratio on natural convection in an annular cavity", International Journal of Numerical Methods for Heat \& Fluid Flow, Vol.3, pp.305-318.

Turan, O., Chakraborty, N., Poole, R.J., (2010), "Laminar natural convection of Bingham fluids in a square enclosure with differentially heated side walls", Journal of Non-Newtonian Fluid Mechanics, Vol.165, pp.901-913.

Turan, O., Chakraborty, N., Poole, R.J., (2012a), "Laminar Rayleigh-Bénard convection of yield stress fluids in a square enclosure", Journal of Non-Newtonian Fluid Mechanics, Vol.171, pp.83-96.

Turan, O., Poole, R.J., Chakraborty, N., (2011a), "Aspect ratio effects in laminar natural convection of Bingham fluids in rectangular enclosures with differentially heated side walls", Journal of NonNewtonian Fluid Mechanics, Vol.166, pp.208-230.

Turan, O., Poole, R.J., Chakraborty, N., (2012b), "Boundary condition effects on natural convection of bingham fluids in a square enclosure with differentially heated horizontal walls", Heat Transfer Engineering, Vol.4, pp.77-97.

Turan, O., Poole, R.J., Chakraborty, N., (2014), "Influences of Boundary Conditions on Laminar Natural Convection of Bingham Fluids in Rectangular Enclosures With Differentially Heated Side Walls", Heat Transfer Engineering, Vol.35, pp.822-849.

Turan, O., Sachdeva, A., Poole, R.J., Chakraborty, N., (2011b), "Laminar Natural Convection of Bingham Fluids in a Square Enclosure with Vertical Walls Subjected to Constant Heat Flux", Numerical Heat Transfer, Part A: Applications, Vol.60, pp.381-409.

Turan, O., Yigit, S., Chakraborty, N., (2017), "Critical condition for Rayleigh-Bénard convection of Bingham fluids in rectangular enclosures", International Communications in Heat and Mass Transfer, Vol.86, pp.117-125.

Vikhansky, A., (2009), "Thermal convection of a viscoplastic liquid with high Rayleigh and Bingham numbers", Physics of Fluids, Vol.21, pp.103103.

Vikhansky, A., (2010), "On the onset of natural convection of Bingham liquid in rectangular enclosures", Journal of Non-Newtonian Fluid Mechanics, Vol.165, pp.1713-1716.

Vola, D., Boscardin, L., Latché, J.C., (2003), "Laminar unsteady flows of Bingham fluids: a numerical strategy and some benchmark results", Journal of Computational Physics, Vol.187, pp.441456.

Yigit, S., Chakraborty, N., (2017a), "Influences of aspect ratio and wall boundary condition on laminar Rayleigh-Bénard convection of Bingham fluids in rectangular enclosures", International Journal of Numerical Methods for Heat \& Fluid Flow, Vol.27, pp.310-333.

Yigit, S., Chakraborty, N., (2017b), "Laminar Natural Convection of Bingham Fluids in Square CrossSectioned Cylindrical Annular Cavity with Differentially Heated Vertical Walls Subjected to Constant Heat Fluxes", Heat Transfer Engineering, Vol.38, pp.1171-1188.

Yigit, S., Chen, S., Quinn, P., Chakraborty, N., (2016), "Numerical investigation of laminar RayleighBénard convection of Bingham fluids in square cross-sectioned cylindrical enclosures", International Journal of Thermal Sciences, Vol.110, pp.356-368. 
Yigit, S., Foxon, T., Chakraborty, N., (2017), "Influences of Boundary Condition on Laminar Natural Convection of Bingham Fluids in Square Cross-Sectioned Cylindrical Annular Enclosures with Differentially Heated Vertical Walls", Heat Transfer Engineering, pp.1-20.

Yigit, S., Poole, R.J., Chakraborty, N., (2015a), "Effects of aspect ratio on natural convection of Bingham fluids in rectangular enclosures with differentially heated horizontal walls heated from below", International Journal of Heat and Mass Transfer, Vol.80, pp.727-736.

Yigit, S., Poole, R.J., Chakraborty, N., (2015b), "Laminar Natural Convection of Bingham Fluids in Inclined Differentially Heated Square Enclosures Subjected to Uniform Wall Temperatures", Journal of Heat Transfer, Vol.137, pp.052504-052504-052512.

Zhang, J., Vola, D., Frigaard, I.A., (2006), "Yield stress effects on Rayleigh-Bénard convection", Journal of Fluid Mechanics, Vol.566, pp.389-419. 
Table 1: Summary of the findings of existing analyses on natural convection of yield stress fluids in enclosed spaces.

\begin{tabular}{|c|c|c|c|c|c|c|c|}
\hline Ref. & Type & Enclosure & $\begin{array}{l}\text { Configuration \& Boundary } \\
\text { conditions }\end{array}$ & $A R=H / L$ & Model \& Fluid & $\mathrm{Ra}, \mathrm{Pr}$ & Correlation \\
\hline $\begin{array}{l}\text { Zhang et al. } \\
\quad(2006)\end{array}$ & $\mathrm{A}, \mathrm{N}$ & Square & $\begin{array}{l}\text { Diff. heated horizontal wall } \\
\text { (CWT) }\end{array}$ & 1 & Bingham & $\begin{array}{c}R a_{\text {crit }} \text { for } \overline{N u}>1 \\
\operatorname{Pr}=1\end{array}$ & - \\
\hline $\begin{array}{l}\text { Balmforth } \\
\text { and Rust } \\
(2009)\end{array}$ & $\mathrm{A}, \mathrm{N}$ & - & $\begin{array}{l}\text { Diff. heated horizontal layers } \\
\qquad(\mathrm{CWT})\end{array}$ & - & $\begin{array}{c}\text { Bingham } \\
\text { Bi-viscosity reg. }\end{array}$ & $R a_{\text {crit }}$ for $\overline{N u}>1$ & - \\
\hline $\begin{array}{l}\text { Turan et al. } \\
\quad(2017)\end{array}$ & $\mathrm{N}$ & Rectangular & $\begin{array}{l}\text { Diff. heated horizontal wall } \\
\text { comparison (CWT-CWHF) }\end{array}$ & $0.25 \leq A R \leq 4$ & Bi-viscosity reg. & $R a_{c r i t}$ for $\overline{N u}>1$ & $R a_{c r i t}=f(B n, \operatorname{Pr}, A R)$ \\
\hline $\begin{array}{l}\text { Vikhansky } \\
\text { (2009) }\end{array}$ & $\mathrm{N}$ & Square & $\begin{array}{l}\text { Diff. heated horizontal wall } \\
\text { (CWT) }\end{array}$ & 1 & Bingham & $R a_{\text {crit }}$ for $\overline{N u}>1$ & - \\
\hline $\begin{array}{l}\text { Vikhansky } \\
\text { (2010) }\end{array}$ & $\mathrm{N}$ & Rectangular & $\begin{array}{l}\text { Diff. heated horizontal wall } \\
\text { (CWT) }\end{array}$ & $0.5 \leq A R \leq 5$ & Bingham & $R a_{\text {crit }}, \begin{array}{c}B n_{\text {crit }} \text { for } \overline{N u}> \\
1\end{array}$ & $B n_{c r i t}=f(B n, A R)$ \\
\hline $\begin{array}{l}\text { Darbouli et } \\
\text { al. (2013) }\end{array}$ & $\mathrm{E}$ & Rectangular & $\begin{array}{l}\text { Diff. heated horizontal wall } \\
\text { (CWT) }\end{array}$ & $6 \leq A R \leq 17.9$ & Carbopol gel & $B n_{\text {crit }}$ for $\overline{N u}>1$ & - \\
\hline $\begin{array}{l}\text { Kebiche et al. } \\
\qquad(2014)\end{array}$ & $\mathrm{E}$ & Rectangular & $\begin{array}{l}\text { Diff. heated horizontal wall } \\
\text { (CWT) }\end{array}$ & 19.3 & Carbopol gel & $B n_{c r i t}$ for $\overline{N u}>1$ & - \\
\hline $\begin{array}{l}\text { Turan et al. } \\
\quad(2010)\end{array}$ & $\mathrm{N}$ & Square & $\begin{array}{l}\text { Diff. heated vertical wall } \\
\text { (CWT) }\end{array}$ & 1 & Bi-viscosity reg. & $\begin{array}{c}10^{3} \leq R a \leq 10^{6} \\
0.1 \leq \operatorname{Pr} \leq 10^{2}\end{array}$ & $\overline{N u}=f(R a, P r, B n)$ \\
\hline $\begin{array}{l}\text { Turan et al. } \\
\quad(2011 \mathrm{~b})\end{array}$ & $\mathrm{N}$ & Square & $\begin{array}{l}\text { Diff. heated vertical wall } \\
\text { comparison (CWT-CWHF) }\end{array}$ & 1 & Bi-viscosity reg. & $\begin{array}{l}10^{3} \leq R a \leq 10^{6} \\
0.1 \leq \operatorname{Pr} \leq 10^{2}\end{array}$ & $\overline{N u}=f(R a, P r, B n)$ \\
\hline
\end{tabular}




\begin{tabular}{|c|c|c|c|c|c|c|c|}
\hline $\begin{array}{l}\text { Turan et al. } \\
\quad(2012 \mathrm{a})\end{array}$ & $\mathrm{N}$ & Square & $\begin{array}{l}\text { Diff. heated horizontal wall } \\
\text { (CWT) }\end{array}$ & 1 & Bi-viscosity reg. & $\begin{array}{l}10^{3} \leq R a \leq 10^{5} \\
0.1 \leq \operatorname{Pr} \leq 10^{2}\end{array}$ & $\overline{N u}=f(R a, P r, B n)$ \\
\hline $\begin{array}{l}\text { Turan et al. } \\
\quad(2012 \mathrm{~b})\end{array}$ & $\mathrm{N}$ & Square & $\begin{array}{l}\text { Diff. heated horizontal wall } \\
\text { comparison (CWT-CWHF) }\end{array}$ & 1 & Bi-viscosity reg. & $\begin{array}{l}10^{3} \leq R a \leq 10^{5} \\
0.1 \leq \operatorname{Pr} \leq 10^{2}\end{array}$ & $\overline{N u}=f(R a, P r, B n)$ \\
\hline $\begin{array}{l}\text { Turan et al. } \\
\quad \text { (2011a) }\end{array}$ & $\mathrm{N}$ & Rectangular & $\begin{array}{l}\text { Diff. heated vertical wall } \\
\text { (CWT) }\end{array}$ & $0.125 \leq A R \leq 8$ & Bi-viscosity reg. & $\begin{array}{c}10^{4} \leq R a \leq 10^{6} \\
\operatorname{Pr}=7\end{array}$ & $\overline{N u}=f(R a, P r, B n, A R)$ \\
\hline $\begin{array}{l}\text { Turan et al. } \\
\quad \text { (2014) }\end{array}$ & $\mathrm{N}$ & Rectangular & $\begin{array}{l}\text { Diff. heated vertical wall } \\
\text { comparison (CWT-CWHF) }\end{array}$ & $0.125 \leq A R \leq 8$ & Bi-viscosity reg. & $\begin{array}{c}10^{4} \leq R a \leq 10^{6} \\
P r=500\end{array}$ & $\overline{N u}=f(R a, \operatorname{Pr}, B n, A R)$ \\
\hline $\begin{array}{l}\text { Yigit et al. } \\
(2015 \mathrm{~b})\end{array}$ & $\mathrm{N}$ & $\begin{array}{c}\text { Square } \\
0^{\circ} \leq \phi \leq 180^{\circ}\end{array}$ & $\begin{array}{l}\text { Diff. heated inclined horizontal } \\
\text { wall (CWT) }\end{array}$ & 1 & Bi-viscosity reg. & $\begin{array}{c}10^{3} \leq R a \leq 10^{5} \\
\operatorname{Pr}=500\end{array}$ & $\overline{N u}=f(R a, P r, B n, \phi)$ \\
\hline $\begin{array}{l}\text { Yigit et al. } \\
\quad(2015 \mathrm{a})\end{array}$ & $\mathrm{N}$ & Rectangular & $\begin{array}{l}\text { Diff. heated horizontal wall } \\
\text { (CWT) }\end{array}$ & $0.25 \leq A R \leq 4$ & Bi-viscosity reg. & $\begin{array}{c}10^{3} \leq R a \leq 10^{5} \\
\operatorname{Pr}=500\end{array}$ & $\overline{N u}=f(R a, P r, B n, A R)$ \\
\hline $\begin{array}{l}\text { Yigit and } \\
\text { Chakraborty } \\
\text { (2017a) }\end{array}$ & $\mathrm{N}$ & Rectangular & $\begin{array}{l}\text { Diff. heated horizontal wall } \\
\text { comparison (CWT-CWHF) }\end{array}$ & $0.25 \leq A R \leq 4$ & Bi-viscosity reg. & $\begin{array}{c}10^{3} \leq R a \leq 10^{5} \\
\operatorname{Pr}=500\end{array}$ & $\overline{N u}=f(R a, P r, B n, A R)$ \\
\hline $\begin{array}{l}\text { Hassan et al. } \\
\quad \text { (2013) }\end{array}$ & $\mathrm{N}$ & $\begin{array}{c}\text { Square } \\
\varepsilon=0.2,0.5,0.8\end{array}$ & $\begin{array}{l}\text { Partially heated horizontal wall } \\
\text { (CWT) }\end{array}$ & 1 & Bi-viscosity reg. & $\begin{array}{c}10^{3} \leq R a \leq 10^{6} \\
\operatorname{Pr}=7\end{array}$ & $\overline{N u}=f(R a, \operatorname{Pr}, B n, \varepsilon)$ \\
\hline $\begin{array}{l}\text { Hassan et al. } \\
\quad \text { (2015) }\end{array}$ & E,N & Square & $\begin{array}{l}\text { Diff. heated horizontal wall } \\
\text { (CWHF) }\end{array}$ & 1 & $\begin{array}{c}\text { Carbopol gel } \\
\text { Herschlel-Bulkley }\end{array}$ & $10^{4} \leq R a \leq 10^{6}$ & $\overline{N u}=f\left(R a, Y_{o}\right)$ \\
\hline $\begin{array}{l}\text { Yigit and } \\
\text { Chakraborty } \\
\text { (2017b) }\end{array}$ & $\mathrm{N}$ & $\begin{array}{l}\text { Cylindrical annular } \\
0.125 \leq r_{i} / L \leq 16\end{array}$ & $\begin{array}{l}\text { Diff. heated vertical wall } \\
\text { (CWHF) }\end{array}$ & 1 & Bi-viscosity reg. & $\begin{array}{c}10^{3} \leq R a \leq 10^{6} \\
10 \leq \operatorname{Pr} \leq 10^{3}\end{array}$ & $\overline{N u}=f\left(R a, \operatorname{Pr}, B n, r_{i} / L\right)$ \\
\hline $\begin{array}{l}\text { Yigit et al. } \\
\quad(2017)\end{array}$ & $\mathrm{N}$ & $\begin{array}{l}\text { Cylindrical annular } \\
0.125 \leq r_{i} / L \leq 16\end{array}$ & $\begin{array}{l}\text { Diff. heated vertical wall } \\
\text { comparison (CWT-CWHF) }\end{array}$ & 1 & Bi-viscosity reg. & $\begin{array}{c}10^{3} \leq R a \leq 10^{6} \\
10 \leq \operatorname{Pr} \leq 10^{3}\end{array}$ & $\overline{N u}=f\left(R a, \operatorname{Pr}, B n, r_{i} / L\right)$ \\
\hline
\end{tabular}


Table 2: Summary of the non-uniform Cartesian meshes used in the current analysis for $0.125 \leq r_{i} / L \leq$ $16,0.125 \leq A R \leq 8$ and $10^{3} \leq R a \leq 10^{6}$ at $\operatorname{Pr}=10^{3}$ with non-dimensional minimum cell distance $\left(\Delta_{\text {min,cell }} / L\right)$ and grid expansion ratio $\left(r_{e}\right)$ values. Numerical uncertainty for the mean Nusselt number on the inner periphery $\overline{N u}_{i}$ for Newtonian (i.e. $B n=0$ ) and Bingham fluids are also shown for different $A R$ at $r_{i} / L=1.0, R a=10^{6}$ and $\operatorname{Pr}=500$ in the case of CWT configuration.

\begin{tabular}{|c|c|c|c|}
\hline \multicolumn{4}{|c|}{$A R=0.125$} \\
\hline Grid & M1 $(120 \times 180)$ & M2 $(160 \times 200)$ & M3 $(200 \times 220)$ \\
\hline$\Delta_{\min , \text { cell }} / L$ & $4.176 \times 10^{-4}$ & $3.135 \times 10^{-4}$ & $2.509 \times 10^{-4}$ \\
\hline$r_{e}$ & 1.013 & 1.01 & 1.008 \\
\hline $\bar{N} \bar{u}_{i}(B n=0)$ & 1.641 & 1.640 & 1.639 \\
\hline$\overline{N u}_{i}(B n=0.2)$ & 1.000 & 1.000 & 1.000 \\
\hline \multicolumn{4}{|c|}{$A R=0.25$} \\
\hline Grid & M1 $(160 \times 180)$ & M2 $(180 \times 220)$ & M3 $(200 \times 240)$ \\
\hline$\Delta_{\min , \text { cell }} / L$ & $6.270 \times 10^{-4}$ & $5.575 \times 10^{-4}$ & $5.018 \times 10^{-4}$ \\
\hline$r_{e}$ & 1.01 & 1.009 & 1.008 \\
\hline $\bar{N}_{\bar{u}}(B n=0)$ & 7.808 & 7.805 & 7.800 \\
\hline$\overline{N u}_{i}(B n=0.2)$ & 1.000 & 1.000 & 1.000 \\
\hline \multicolumn{4}{|c|}{$A R=0.5$} \\
\hline Grid & M1 $(180 \times 220)$ & M2 $(200 \times 240)$ & M3 $(220 \times 260)$ \\
\hline$\Delta_{\min , \text { cell }} / L$ & $1.115 \times 10^{-3}$ & $1.003 \times 10^{-3}$ & $9.127 \times 10^{-4}$ \\
\hline$r_{e}$ & 1.009 & 1.008 & 1.007 \\
\hline $\bar{N} \bar{u}_{i}(B n=0)$ & 12.503 & 12.501 & 12.499 \\
\hline$\overline{N u}_{i}(B n=0.2)$ & 5.880 & 5.850 & 5.810 \\
\hline \multicolumn{4}{|c|}{$A R=1.0$} \\
\hline Grid & M1 $(180 \times 180)$ & M2 $(240 \times 240)$ & M3 $(260 \times 260)$ \\
\hline$\Delta_{\min , \text { cell }} / L$ & $2.230 \times 10^{-3}$ & $1.673 \times 10^{-3}$ & $1.545 \times 10^{-3}$ \\
\hline$r_{e}$ & 1.009 & 1.006 & 1.006 \\
\hline $\bar{N}_{\bar{u}}(B n=0)$ & 12.421 & 12.419 & 12.413 \\
\hline$\overline{N u}_{i}(B n=0.2)$ & 8.742 & 8.740 & 8.736 \\
\hline \multicolumn{4}{|c|}{$A R=2.0$} \\
\hline Grid & M1 $(140 \times 300)$ & M2 $(160 \times 320)$ & M3 $(200 \times 400)$ \\
\hline$\Delta_{\min , \text { cell }} / L$ & $2.678 \times 10^{-3}$ & $2.508 \times 10^{-3}$ & $2.007 \times 10^{-3}$ \\
\hline$r_{e}$ & 1.01 & 1.01 & 1.008 \\
\hline $\bar{N}_{\bar{u}}(B n=0)$ & 11.234 & 11.230 & 11.200 \\
\hline$\overline{N u}_{i}(B n=0.2)$ & 8.849 & 8.845 & 8.840 \\
\hline \multicolumn{4}{|c|}{$A R=4.0$} \\
\hline Grid & M1 $(140 \times 400)$ & M2 $(160 \times 480)$ & M3 $(200 \times 600)$ \\
\hline$\Delta_{\min , \text { cell }} / L$ & $2.865 \times 10^{-3}$ & $2.508 \times 10^{-3}$ & $2.007 \times 10^{-3}$ \\
\hline$r_{e}$ & 1.01 & 1.01 & 1.008 \\
\hline $\bar{N} \bar{u}_{i}(B n=0)$ & 9.766 & 9.763 & 9.760 \\
\hline$\overline{N u}_{i}(B n=0.2)$ & 8.160 & 8.157 & 8.155 \\
\hline \multicolumn{4}{|c|}{$A R=8.0$} \\
\hline Grid & M1 $(140 \times 480)$ & M2 $(160 \times 540)$ & M3 $(180 \times 560)$ \\
\hline$\Delta_{\min , \text { cell }} / L$ & $2.865 \times 10^{-3}$ & $2.508 \times 10^{-3}$ & $2.230 \times 10^{-3}$ \\
\hline$r_{e}$ & 1.01 & 1.009 & 1.009 \\
\hline $\bar{N} \bar{u}_{i}(B n=0)$ & 8.388 & 8.385 & 8.380 \\
\hline$\overline{N u}_{i}(B n=0.2)$ & 7.231 & 7.225 & 7.220 \\
\hline
\end{tabular}


Table 3: Summary of $\left(B n_{\max }^{*}\right)_{\operatorname{Rec}}$ correlation given by Eq. (36).

\begin{tabular}{|c|c|}
\hline \multicolumn{2}{|c|}{$\left(B n_{\text {max }}^{*}\right)_{R e c}=c_{1} R a^{0.31} \operatorname{Pr}^{-0.21} A R^{-0.25}$} \\
\hline \multirow{2}{*}{ CWT } & $c_{1}=\left[\frac{1-0.062 \ln R a}{90.5-5.117 \ln R a}\right]+[0.021-0.001 \ln R a] \operatorname{erf}[2 A R-1.5]$ for $A R \leq 1$ \\
\cline { 2 - 3 } & $c_{1}=\left[\frac{1-0.052 \ln R a}{85.34-3.742 \ln R a}\right]+[0.024-0.001 \ln R a] \operatorname{erf}[2 A R-3.352]$ for $A R>1$ \\
\hline \multirow{2}{*}{ CWHF } & $\left(B n_{\text {max }}^{*}\right)_{\operatorname{Rec}}=c_{2} \operatorname{Ra}^{0.31} \operatorname{Pr}^{-0.21} A R^{-0.25}$ \\
\cline { 2 - 3 } & $c_{2}=\left[\frac{1-0.054 \ln R a}{94.696-4 \ln R a}\right]+[0.0175-0.0008 \ln R a] \operatorname{erf}[2 A R-1.402]$ for $A R \leq 1$ \\
\hline
\end{tabular}


Table 4: Summary of $\left(B n_{\max }\right)_{i}$ correlations given by Eqs. (37) and (38) for CWT and CWHF boundary conditions respectively.

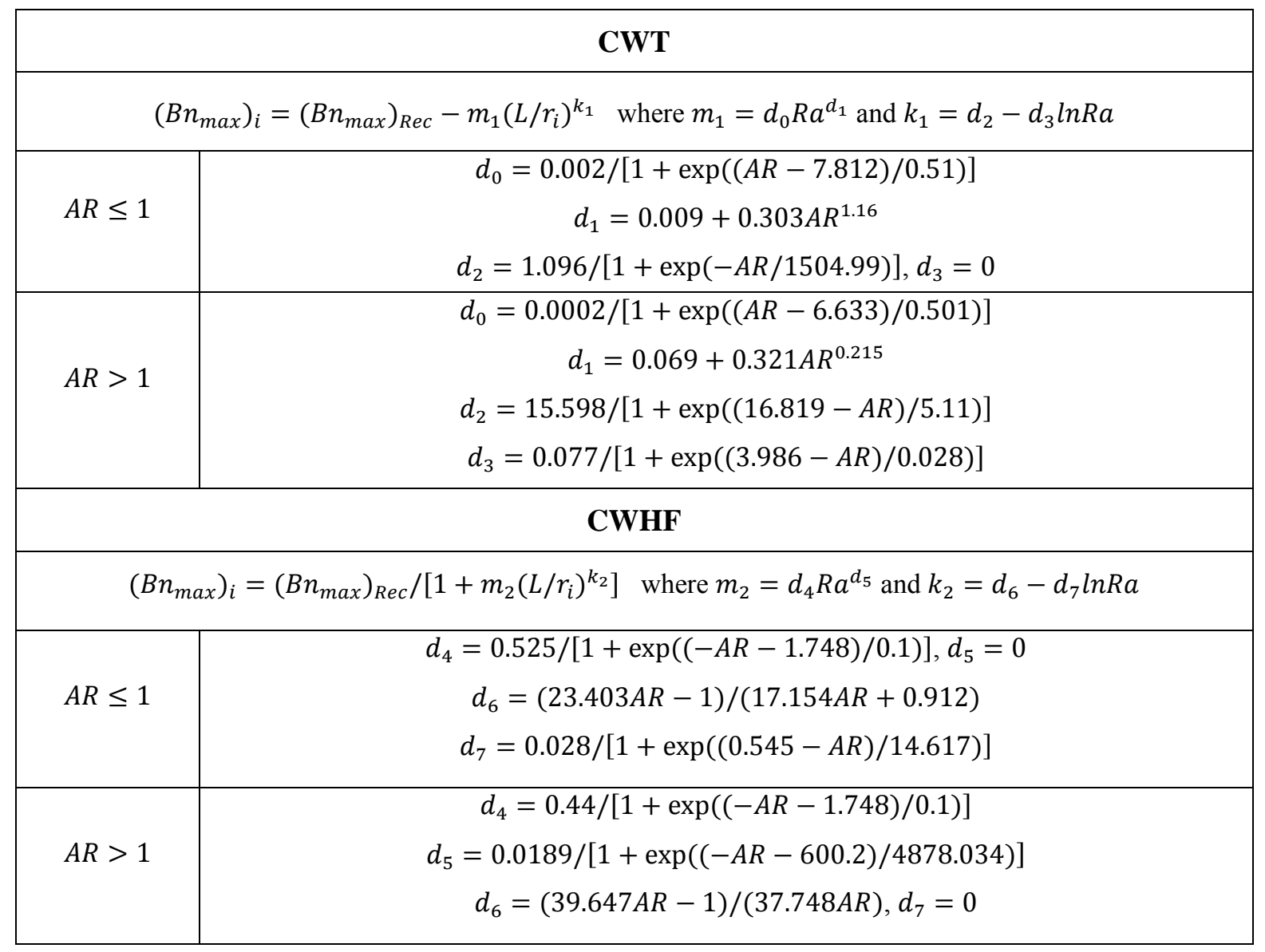


Table 5: Summary of $\overline{N u}_{i} /\left(\overline{N u}_{i}\right)_{\text {cond }}$ correlation for rectangular cross-sectional cylindrical annular enclosures for CWT and CWHF boundary conditions respectively.

\begin{tabular}{|c|c|}
\hline & CWT \\
\hline$\frac{\left[\overline{N u}_{i} /\left(\overline{N u}_{i}\right)_{c c}\right.}{\left[(\overline{N u})_{B n=0} /(\overline{N u}\right.}$ & $\begin{array}{l}\left\{\left[1-\left(\frac{1}{4.55}\right)^{-2} B n^{* *}\right]^{2 / n_{1}}+\left[\frac{2\left[1-\left(B n^{*} / B n_{\max }^{*}\right)^{\left(b_{0}\right)_{i}}\right]^{\left(b_{1}\right)}}{B n^{*}+\sqrt{B n^{* 2}+4}}\right]^{1 / n_{1}}\right\}^{n_{1}} \text { for } \overline{N \bar{u}_{i}} /\left(\overline{N u}_{i}\right)_{\text {cond }}>1 \\
\overline{N u}_{i} /\left(\overline{N u}_{i}\right)_{\text {cond }}=1 \text { is when }\left(\overline{N u}_{i}\right)_{B n=0} /\left(\overline{N u}_{i}\right)_{\text {cond }}=1 \\
\left.+k_{3}\left(L / r_{i}\right)^{0.01}\right] \text { and }\left(b_{1}\right)_{i}=\left(0.676 R a_{C W T}{ }^{0.131} \operatorname{Pr}^{0.11}\right) /\left[1+k_{4}\left(L / r_{i}\right)^{0.01}\right]\end{array}$ \\
\hline $0.5<A R \leq 1$ & $\begin{array}{c}k_{3}=[1.1-1.018 A R] R a^{0.208 A R-0.059} \\
k_{4}=[0.607-0.147 A R] \ln R a-[1.79+0.6 A R]\end{array}$ \\
\hline $1<A R \leq 8$ & $\begin{array}{c}k_{3}=[0.137 /(1+\exp ((A R-2.326) / 0.33))] R a^{0.067 A R^{1.45}} \\
k_{4}=\left(0.513 A R^{0.26}\right) \ln R a-\left(44.95 A R^{0.064}-44.52\right)\end{array}$ \\
\hline \multicolumn{2}{|r|}{ CWHF } \\
\hline $\begin{array}{r}\frac{\left[N u_{i} /\left(\overline{N u}_{i}\right)_{c}\right.}{\left[\left(\overline{N u_{i}}\right)_{B n=0} /(\overline{N \imath}\right.} \\
\\
\quad\left(b_{2}\right)_{i}\end{array}$ & $\begin{array}{l}\left\{\left[1-\left(\frac{1}{5.5}\right)^{-2} B n^{* *}\right]^{2 / n_{2}}+\left[\frac{2\left[1-\left(B n^{*} / B n_{\max }^{*}\right)^{\left(b_{2}\right)_{i}}\right]^{\left(b_{3}\right)_{i}}}{B n^{*}+\sqrt{B n^{* 2}+4}}\right]^{1 / n_{2}}\right\}^{n_{2}} \text { for } \overline{N u}_{i} /\left(\overline{N u}_{i}\right)_{\text {cond }} \\
\overline{N u}_{i} /\left(\overline{N u}_{i}\right)_{c o n d}=1 \text { is when }\left(\overline{N u}_{i}\right)_{B n=0} /\left(\overline{N u}_{i}\right)_{c o n d}=1 \\
\left.+k_{5}\left(L / r_{i}\right)^{0.01}\right] \text { and }\left(b_{3}\right)_{i}=\left(0.25 R a_{C W H F}{ }^{0.207} \operatorname{Pr}^{0.062}\right) /\left[1+k_{6}\left(L / r_{i}\right)^{0.01}\right]\end{array}$ \\
\hline $0.5 \leq A R \leq 8$ & $k_{5}=[0.168 /(1+\exp ((14.1-A R) / 2.282))] R a^{06-0.028 A R}$ \\
\hline $0.5 \leq A R \leq 1$ & $k_{6}=\left[0.009 A R^{0.585}\right] R a^{0.542-0.1 A R}$ \\
\hline $1<A R \leq 8$ & $k_{6}=\left[\frac{1-0.479 A R}{318.7-157.9 A R}\right](\ln R a)^{n_{3}}$ and $n_{3}=[2.764 /(1+\exp ((1.49-A R) / 0.671))]$ \\
\hline
\end{tabular}




\section{FIGURE CAPTIONS}

Fig. 1: Schematic diagram of the simulation domain: a) CWT, b) CWHF configurations.

Fig. 2: Variations of non-dimensional temperature $\theta$ and non-dimensional axial (radial) $W=w L / \alpha$ $(U=u L / \alpha)$ velocity components along the horizontal (vertical) mid-plane for different $R a$ values at $r_{i} / L=1, A R=0.5$ and $\operatorname{Pr}=500$ for Newtonian (i.e. $B n=0$ ) and Bingham fluids. The pure conduction solution is shown by triangles (circles) for the CWT (CWHF) configuration.

Fig. 3: Contours of non-dimensional temperature $\theta$ and non-dimensional stream function $(\Psi=\psi / \alpha)$ for different $R a$ values at $r_{i} / L=1, A R=0.5$ and $P r=500$ for Newtonian (i.e. $B n=0$ ) and Bingham fluid $(B n=0.05)$ cases for the CWT configuration. The apparently unyielded regions (AURs) are shown by grey shading.

Fig. 4: Variations of non-dimensional temperature $\theta$ and non-dimensional axial (radial) $W(U)$ velocity components along the horizontal (vertical) mid-plane for different $B n$ values at $r_{i} / L=1, R a=$ $10^{6}$ and $\operatorname{Pr}=500$ for $A R=0.5$ and 2 . The pure conduction solution is shown by triangles (circles) for the CWT (CWHF) configuration.

Fig. 5: Contours of $\theta$ and $\Psi$ with AURs for different $B n$ values at $r_{i} / L=1, A R=0.5, R a=$ $10^{6}$ and $\operatorname{Pr}=500$ for the CWT configuration.

Fig. 6: Variations of non-dimensional temperature $\theta$ and non-dimensional axial (radial) $W(U)$ velocity components along the horizontal (vertical) mid-plane for different $A R$ values at $r_{i} / L=1, R a=10^{6}$, $B n=0.2$ and $\operatorname{Pr}=500$. The pure conduction solution is shown by triangles (circles) for the CWT (CWHF) configurations. The $A R$ values indicated by an asterisk in the legend represent the aspect ratio at which the highest $\overline{N u}_{i} /\left(\bar{N}_{i}\right)_{\text {cond }}$ is obtained as shown in Fig. 7.

Fig. 7: a)Variations of $\bar{N}_{i} / N u_{\text {cond }}$ with $A R$ for different values of $R a$ at $r_{i} / L=1$ and $\operatorname{Pr}=500$ for Newtonian fluids (i.e. $B n=0$ ) for both CWT and CWHF configurations, b) Variations of $\overline{N u}_{i} /\left(\overline{N u}_{i}\right)_{\text {cond }}$ with $B n$ for different values of $A R$ at $r_{i} / L=1, R a=10^{6}$ and $\operatorname{Pr}=500$ for both CWT and CWHF configurations.

Fig 8: Contours of non-dimensional stream function $\Psi$ with AURs for different $A R$ values at $r_{i} / L=$ 1, $R a=10^{6}, B n=0.2$ and $\operatorname{Pr}=500$ for CWHF boundary condition.

Fig. 9: Variations of $\theta$ and $W(U)$ along the horizontal (vertical) mid-plane for different values of $r_{i} / L$ for $A R=0.5, R a=10^{6}, B n=0.1$ and $P r=500$ for both CWT and CWHF configurations. The pure conduction solutions for $\theta$ are shown by the dashed lines.

Fig. 10: Variations of $\overline{N u}_{i} /\left(\overline{N u}_{i}\right)_{\text {cond }}$ with $r_{i} / L$ for different values of $B n$ for $A R=0.5, R a=10^{6}$ at $P r=500$ for both CWT and CWHF configurations. The dashed lines represent corresponding value of mean Nusselt number for rectangular enclosures $\overline{N u}_{R e c}$ for each $B n$ values.

Fig. 11: The variations of $\overline{N u}_{i} /\left(\overline{N u}_{i}\right)_{\text {cond }}$ with $r_{i} / L$ for Newtonian fluids (i.e. $B n=0$ ) for different values of $R a$ and $A R:$ a) 0.25 , b) 1, c) 4, d) 8 at $P r=500$ along with predictions of Eq. 32 (solid line). The corresponding values for the $\overline{N u}_{R e c}$ are shown by the dashed line. 
Fig. 12: The variations of $\left(B n_{\max }\right)_{i}$ with $r_{i} / L$ for different values of $R a$ and $A R:$ a) 0.5, b) 1 , c) 4 , d) 8 at $\operatorname{Pr}=500$ along with the predictions of Eq. (37) and (38) (solid line) for CWT and CWHF respectively. The corresponding values for the $\left(B n_{\max }\right)_{\operatorname{Rec}}$ are shown by the dashed line (Eq. (36)).

Fig 13: The variations of $\overline{N u}_{i} /\left(\overline{N u}_{i}\right)_{\text {cond }}$ with $B n$ for different values of $A R$ and $r_{i} / L$ : a) 0.125, b) 1 , c) 4, d) 16 at $R a=10^{6}$ and $\mathrm{Pr}=500$ along with predictions of the correlation listed in Table 5 for CWT and CWHF configuration respectively. 


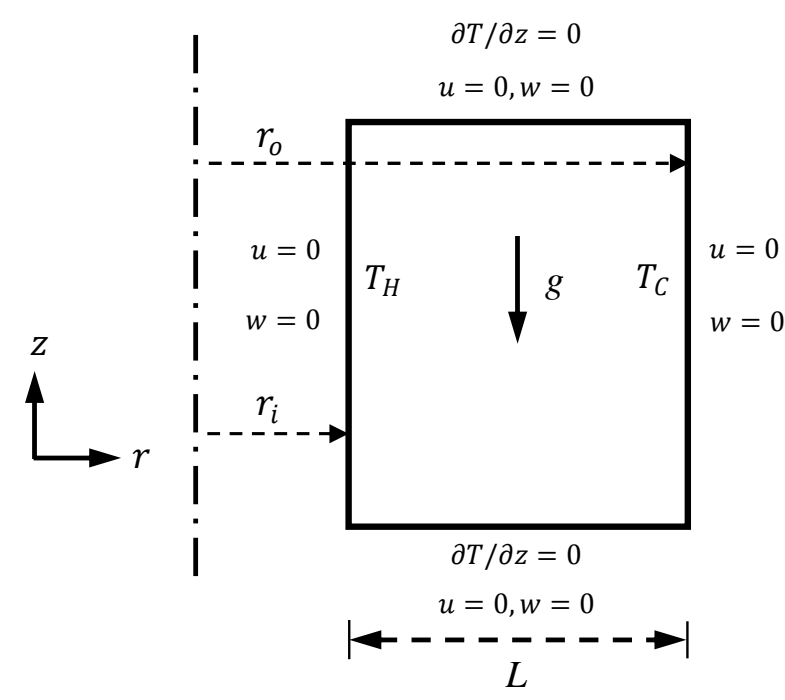

a)

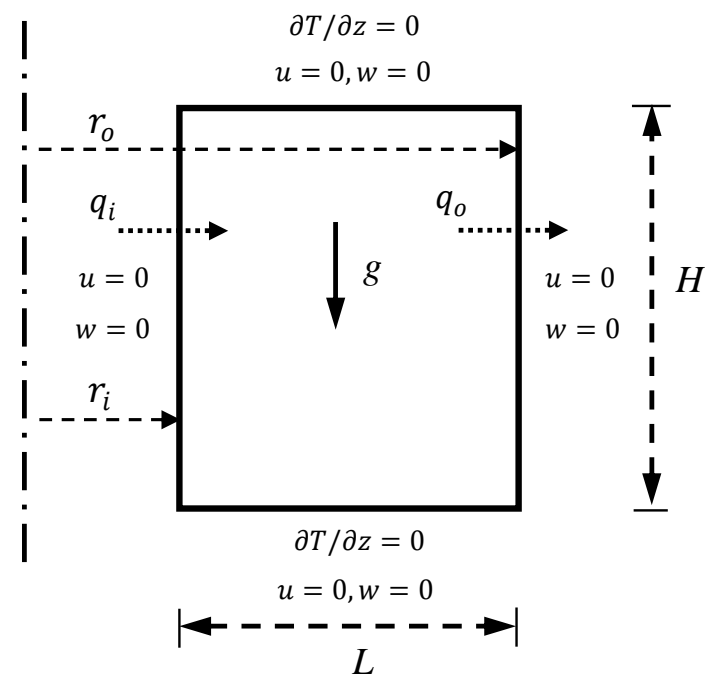

b)

Fig. 1: Schematic diagram of the simulation domain: a) CWT, b) CWHF configurations. 

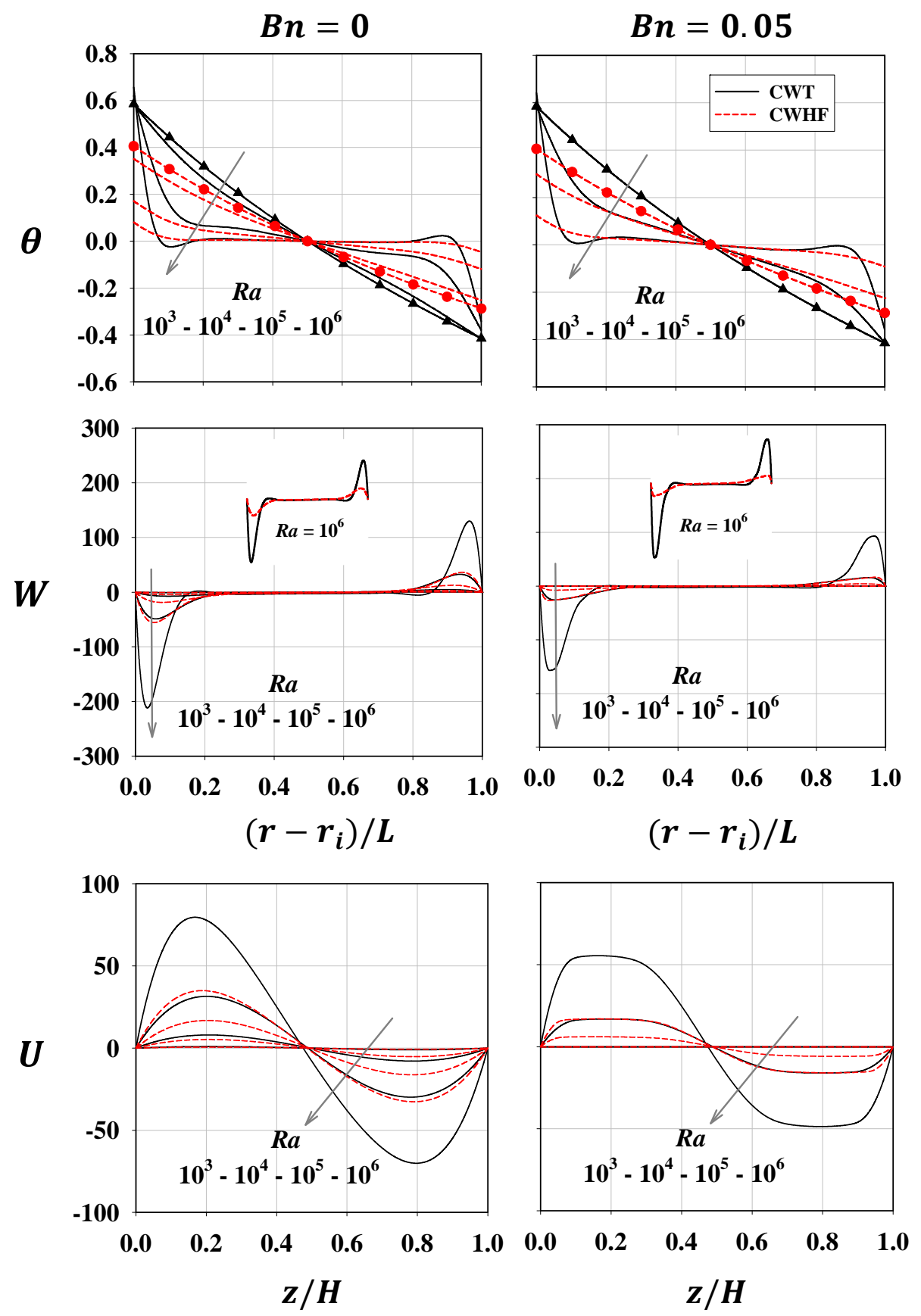

Fig. 2: Variations of non-dimensional temperature $\theta$ and non-dimensional axial (radial) $W=w L / \alpha$ $(U=u L / \alpha)$ velocity components along the horizontal (vertical) mid-plane for different $R a$ values at $r_{i} / L=1, A R=0.5$ and $\operatorname{Pr}=500$ for Newtonian (i.e. $B n=0$ ) and Bingham fluids. The pure conduction solution is shown by triangles (circles) for the CWT (CWHF) configuration. 


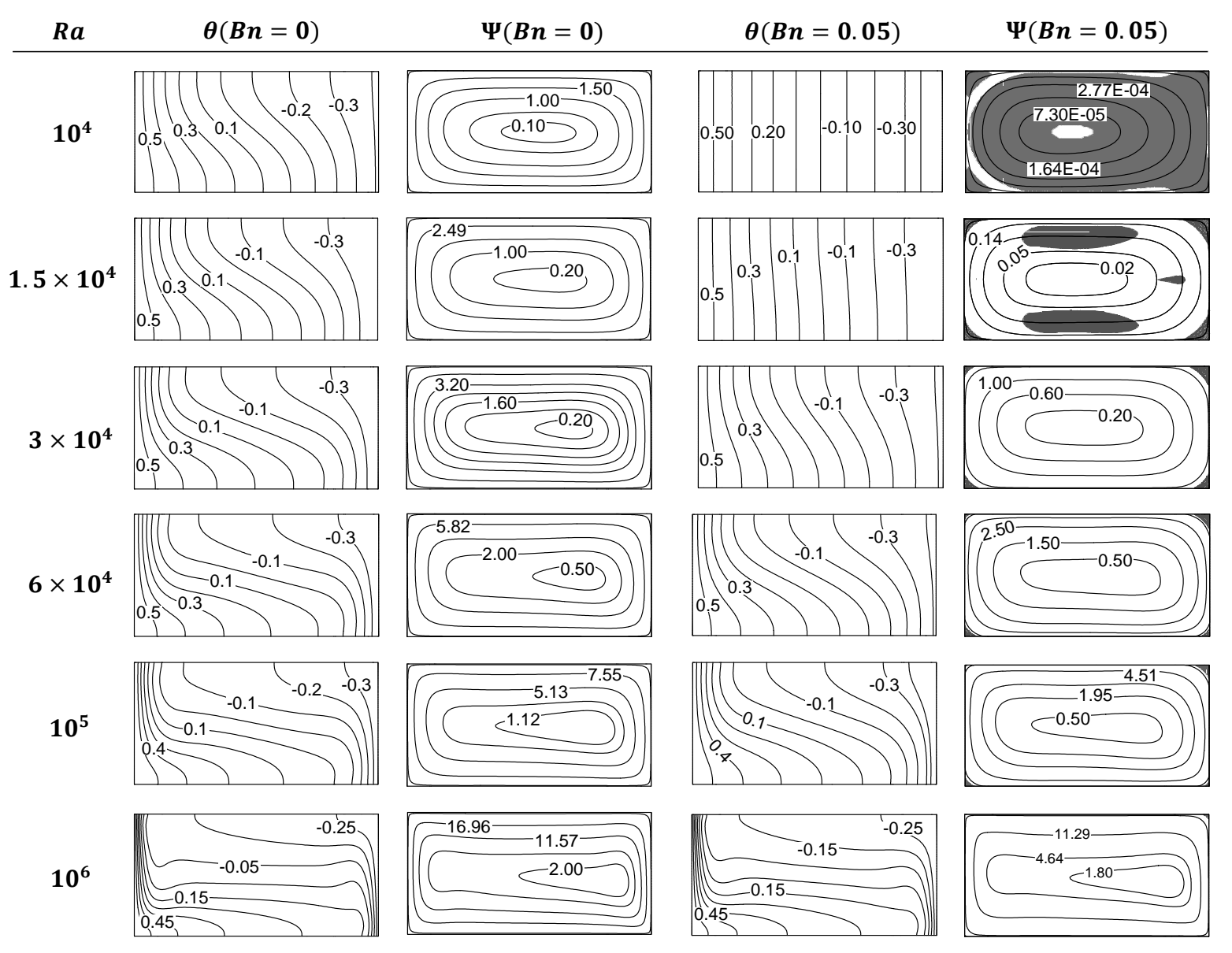

Fig. 3: Contours of non-dimensional temperature $\theta$ and non-dimensional stream function $(\Psi=\psi / \alpha)$ for different $R a$ values at $r_{i} / L=1, A R=0.5$ and $P r=500$ for Newtonian (i.e. $B n=0$ ) and Bingham fluid $(B n=0.05)$ cases for the CWT configuration. The apparently unyielded regions (AURs) are shown by grey shading. 

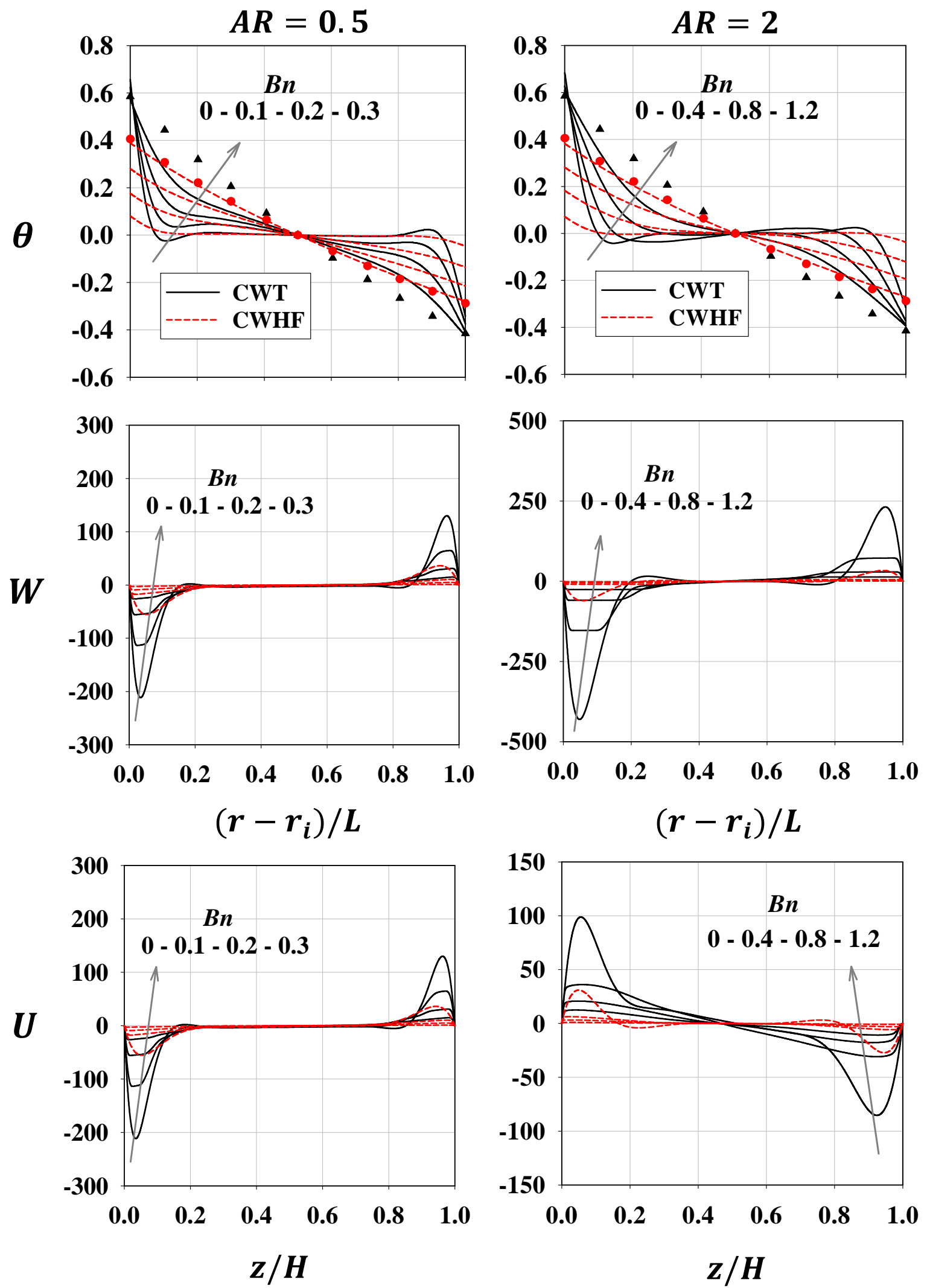

Fig. 4: Variations of non-dimensional temperature $\theta$ and non-dimensional axial (radial) $W(U)$ velocity components along the horizontal (vertical) mid-plane for different $B n$ values at $r_{i} / L=1, R a=$ $10^{6}$ and $\operatorname{Pr}=500$ for $A R=0.5$ and 2. The pure conduction solution is shown by triangles (circles) for the CWT (CWHF) configuration. 


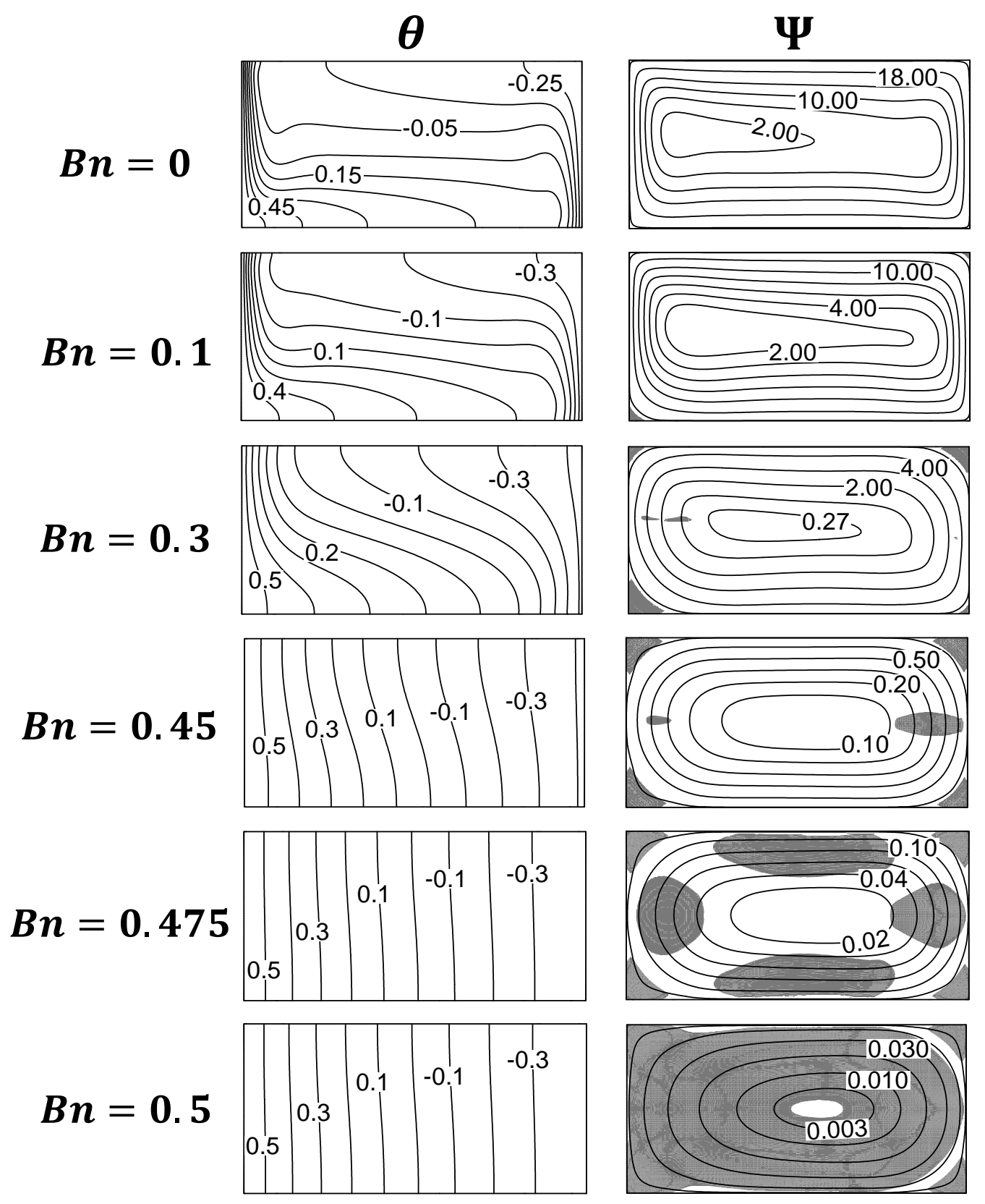

Fig. 5: Contours of $\theta$ and $\Psi$ with AURs for different $B n$ values at $r_{i} / L=1, A R=0.5, R a=$ $10^{6}$ and $\operatorname{Pr}=500$ for the CWT configuration. 

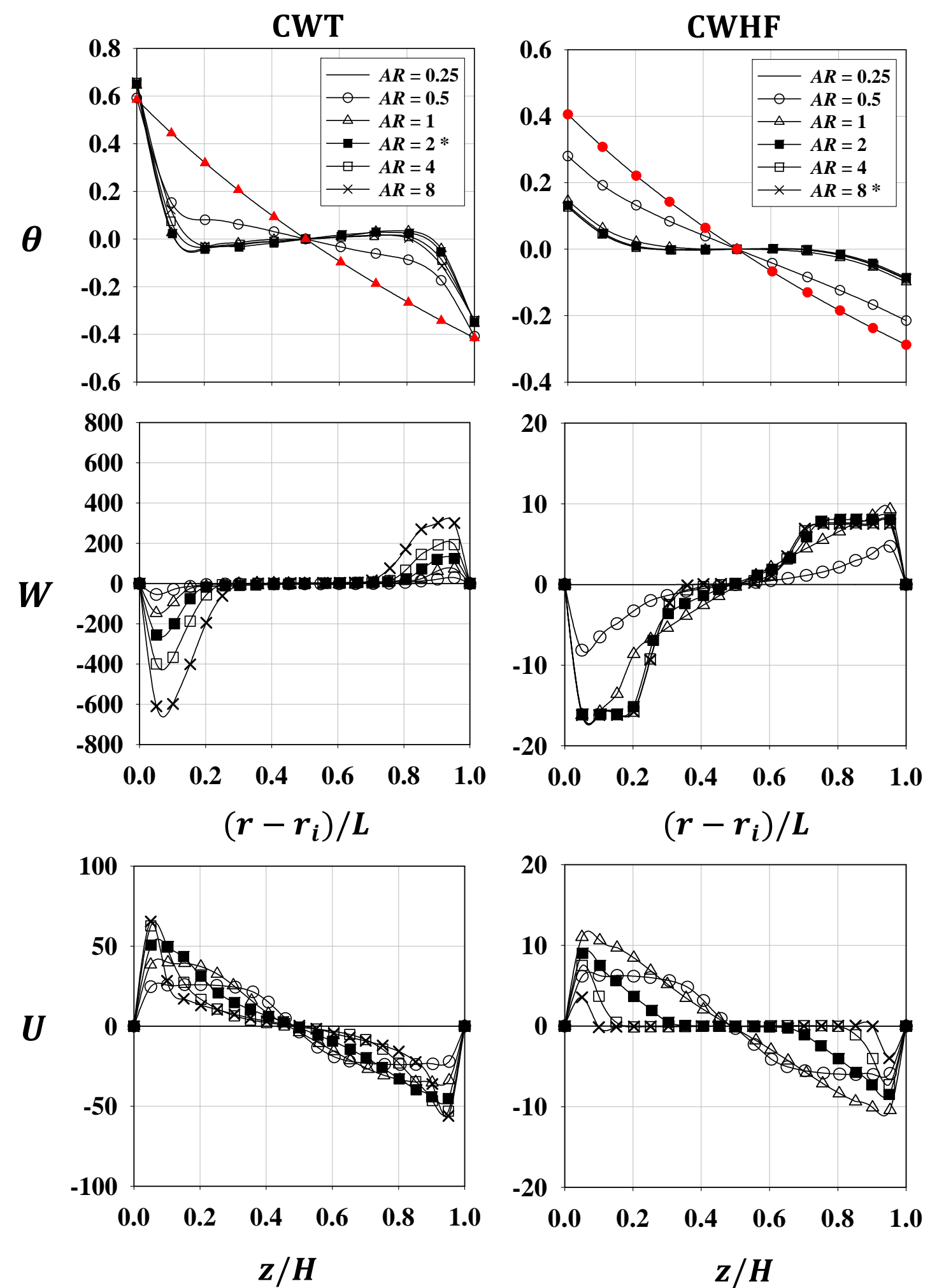

Fig. 6: Variations of non-dimensional temperature $\theta$ and non-dimensional axial (radial) $W(U)$ velocity components along the horizontal (vertical) mid-plane for different $A R$ values at $r_{i} / L=1, R a=10^{6}$, $B n=0.2$ and $\operatorname{Pr}=500$. The pure conduction solution is shown by triangles (circles) for the CWT (CWHF) configurations. The $A R$ values indicated by an asterisk in the legend represent the aspect ratio at which the highest $\overline{N u}_{i} /\left(\bar{N}_{i}\right)_{\text {cond }}$ is obtained as shown in Fig. 7. 

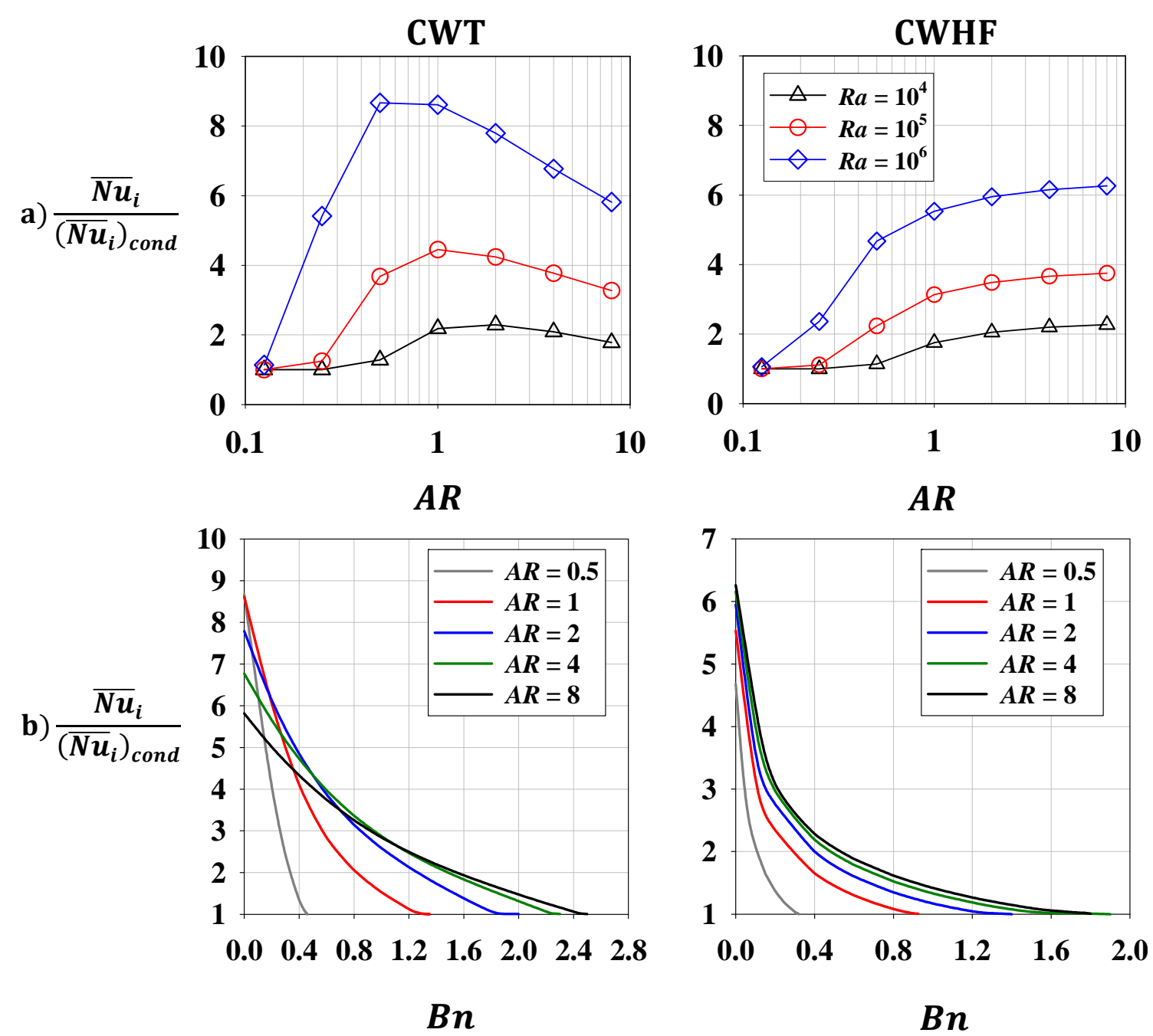

Fig. 7: a)Variations of $\overline{N u}_{i} / N u_{\text {cond }}$ with $A R$ for different values of $R a$ at $r_{i} / L=1$ and $P r=500$ for Newtonian fluids (i.e. $B n=0$ ) for both CWT and CWHF configurations, b) Variations of $\overline{N u}_{i} /\left(\overline{N u}_{i}\right)_{\text {cond }}$ with $B n$ for different values of $A R$ at $r_{i} / L=1, R a=10^{6}$ and $\operatorname{Pr}=500$ for both CWT and CWHF configurations. 


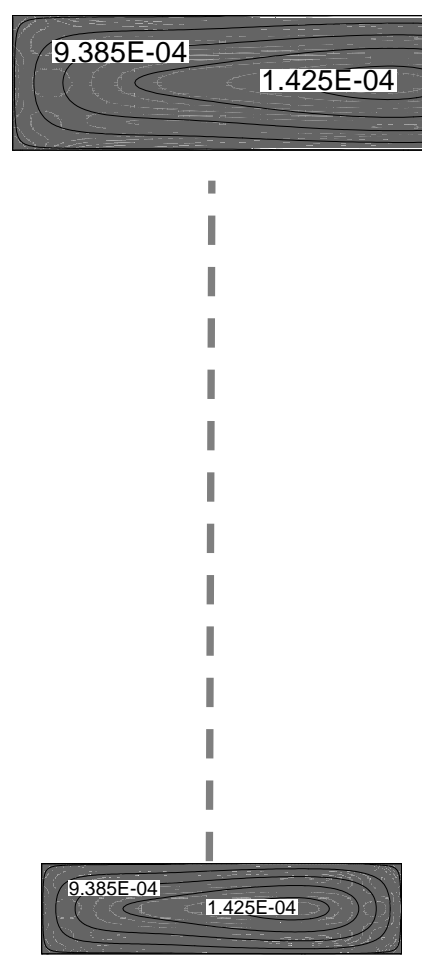

$$
A R=0.25
$$

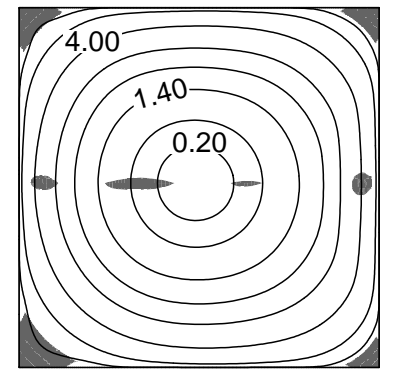

$A R=1.0$

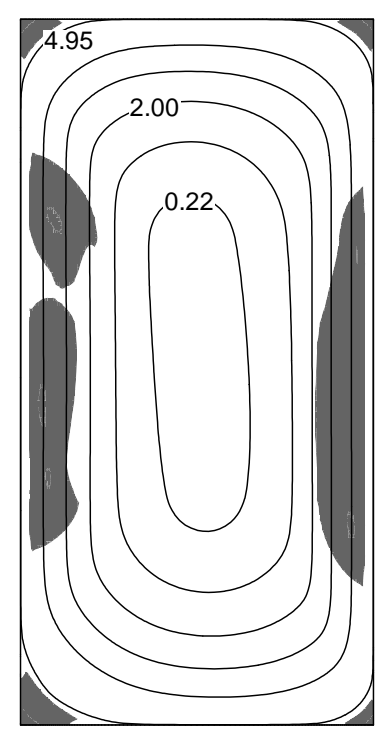

$A R=2.0$

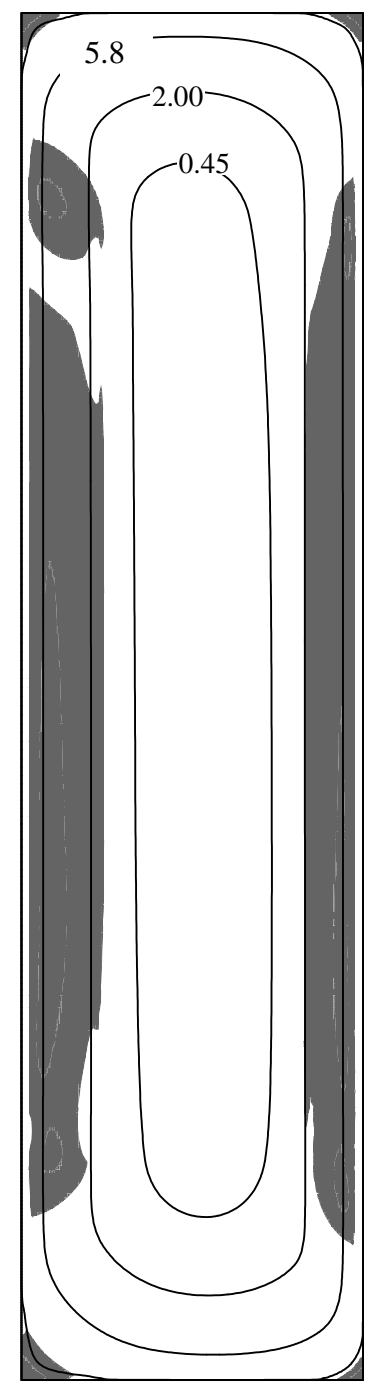

$A R=4.0$

Fig 8: Contours of non-dimensional stream function $\Psi$ with AURs for different $A R$ values at $r_{i} / L=$ 1, $R a=10^{6}, B n=0.2$ and $P r=500$ for CWHF boundary condition. 

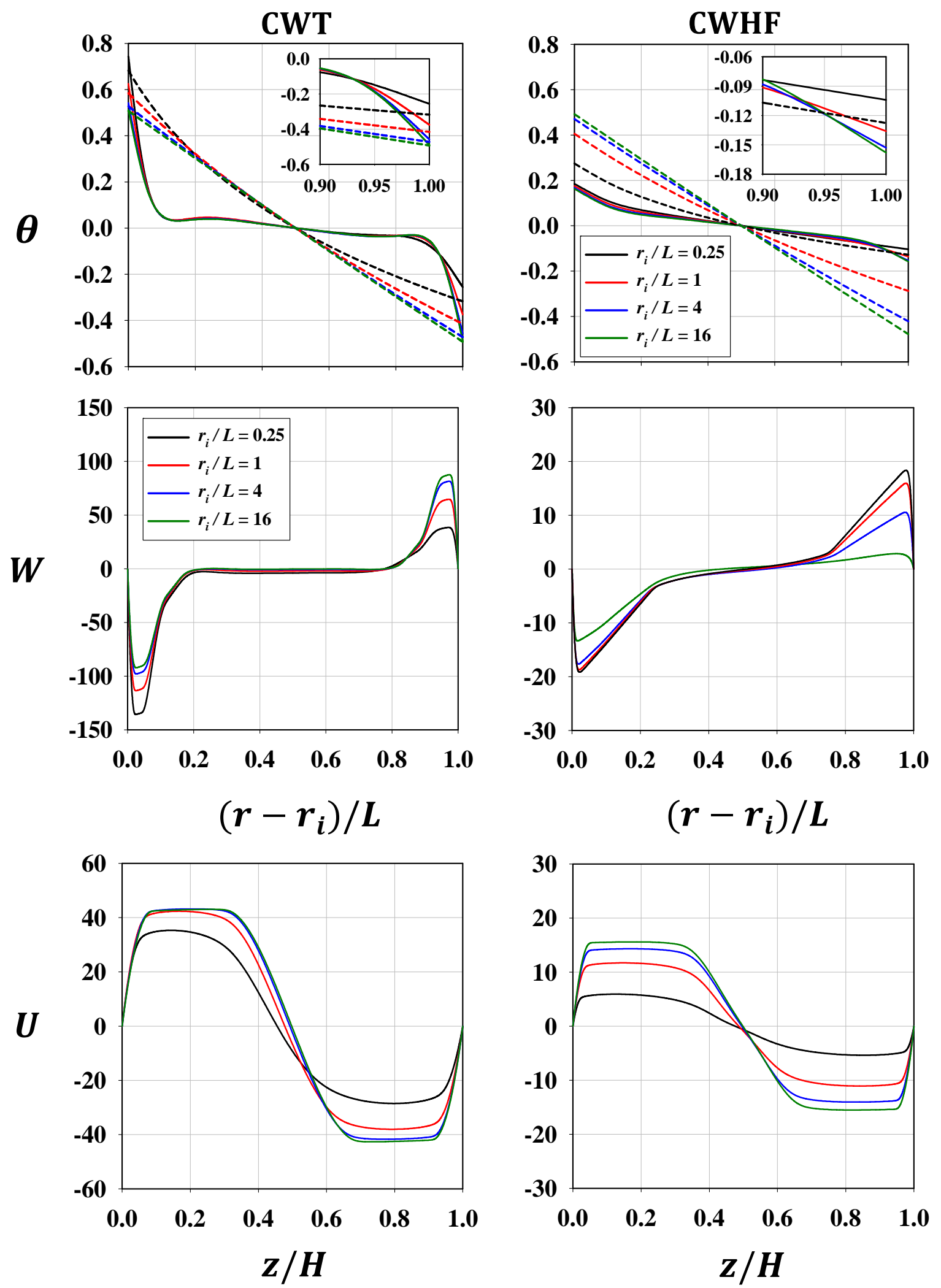

Fig. 9: Variations of $\theta$ and $W(U)$ along the horizontal (vertical) mid-plane for different values of $r_{i} / L$ for $A R=0.5, R a=10^{6}, B n=0.1$ and $P r=500$ for both CWT and CWHF configurations. The pure conduction solutions for $\theta$ are shown by the dashed lines. 

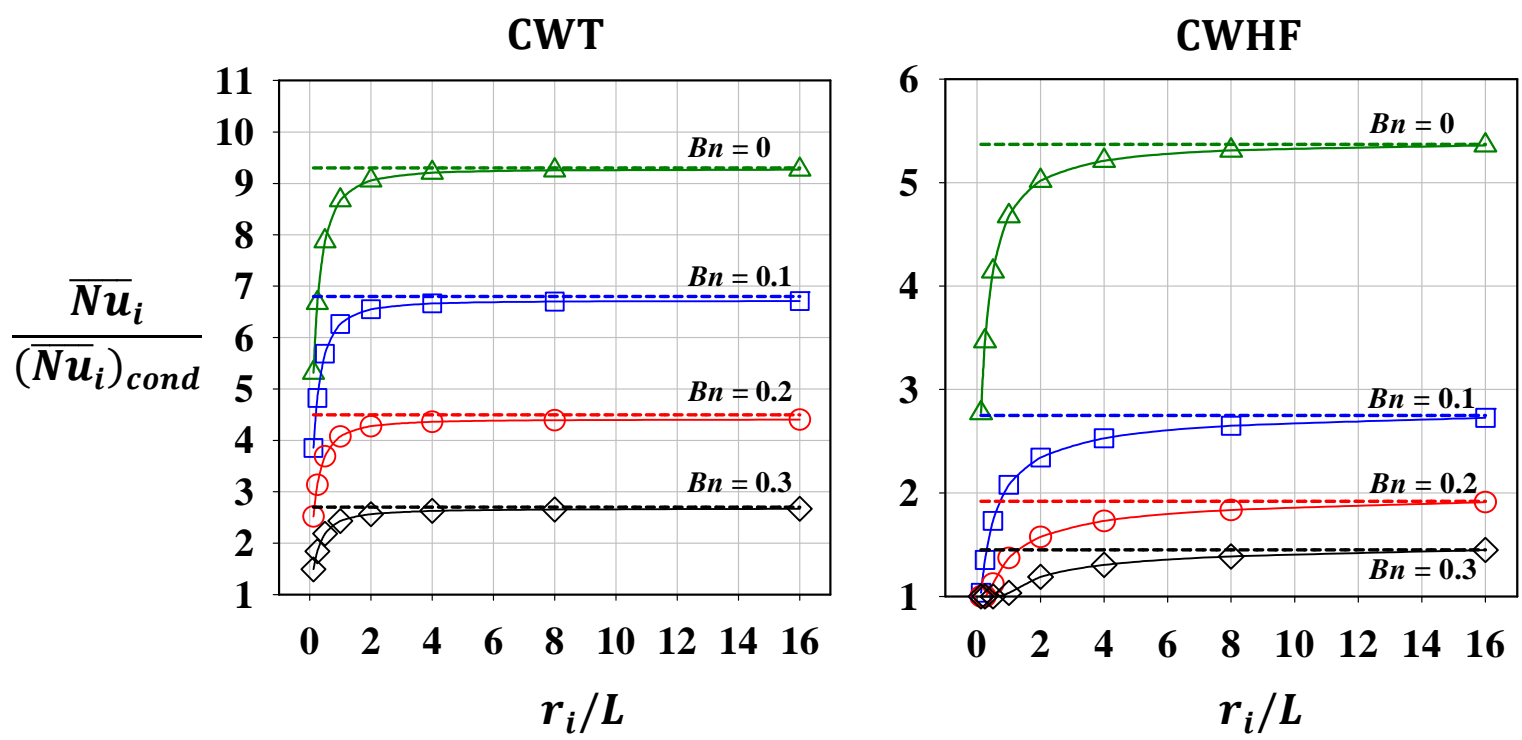

Fig. 10: Variations of $\overline{N u}_{i} /\left(\overline{N u}_{i}\right)_{\text {cond }}$ with $r_{i} / L$ for different values of $B n$ for $A R=0.5, R a=10^{6}$ at $P r=500$ for both CWT and CWHF configurations. The dashed lines represent corresponding value of mean Nusselt number for rectangular enclosures $\overline{N u}_{R e c}$ for each $B n$ values. 


\section{CWT}

a) $\frac{\overline{N u}_{i}}{\left(\overline{N u}_{i}\right)_{c o n d}}$

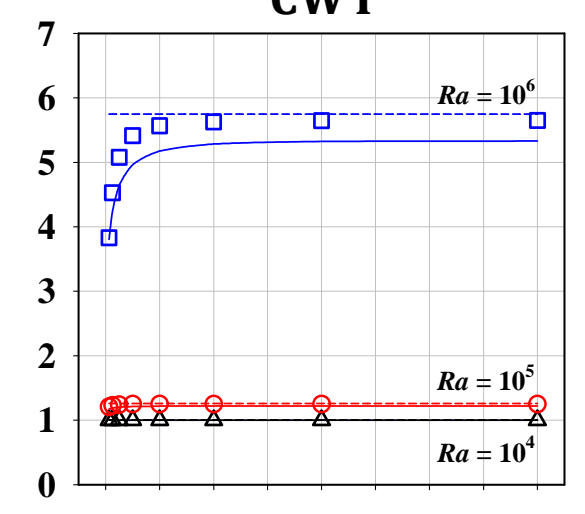

b) $\frac{\overline{\boldsymbol{N u}}_{i}}{\left(\overline{\boldsymbol{N u}}_{i}\right)_{\text {cond }}}$

c) $\frac{\overline{\boldsymbol{N u}}_{i}}{\left(\overline{\boldsymbol{N u}}_{i}\right)_{\text {cond }}}$

d) $\frac{\overline{\boldsymbol{N u}}_{i}}{\left(\overline{\boldsymbol{N u}}_{i}\right)_{\text {cond }}}$

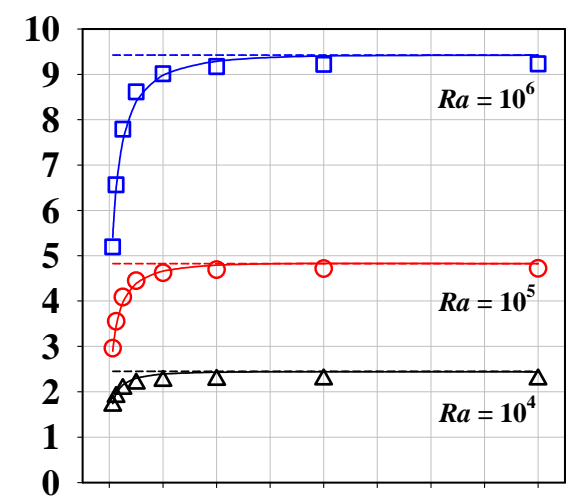

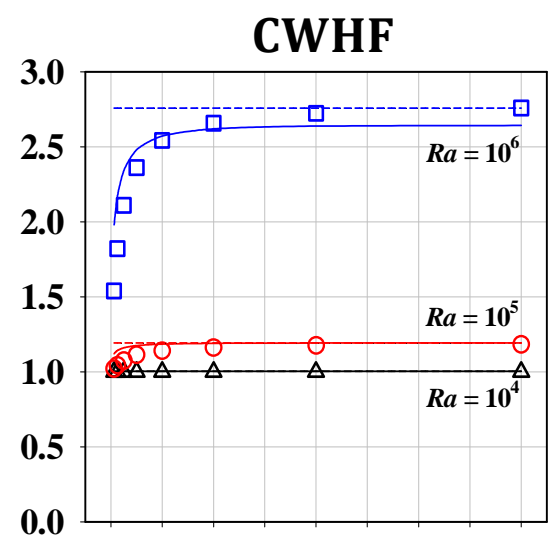
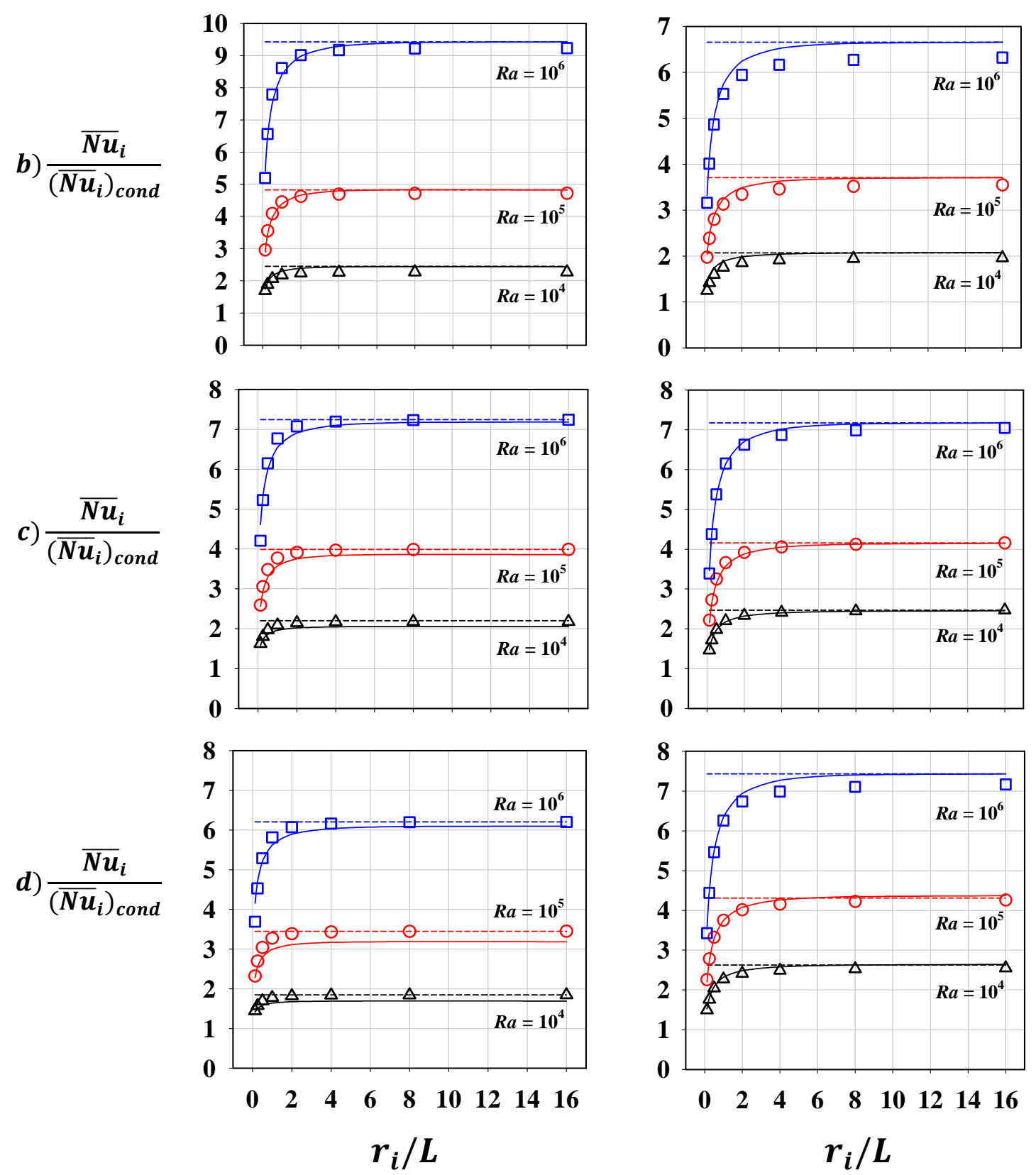

Fig. 11: The variations of $\overline{N u}_{i} /\left(\overline{N u}_{i}\right)_{\text {cond }}$ with $r_{i} / L$ for Newtonian fluids (i.e. $\left.B n=0\right)$ for different values of $R a$ and $A R:$ a) 0.25 , b) 1, c) 4, d) 8 at $P r=500$ along with predictions of Eq. 32 (solid line). The corresponding values for the $\overline{N u}_{R e c}$ are shown by the dashed line. 


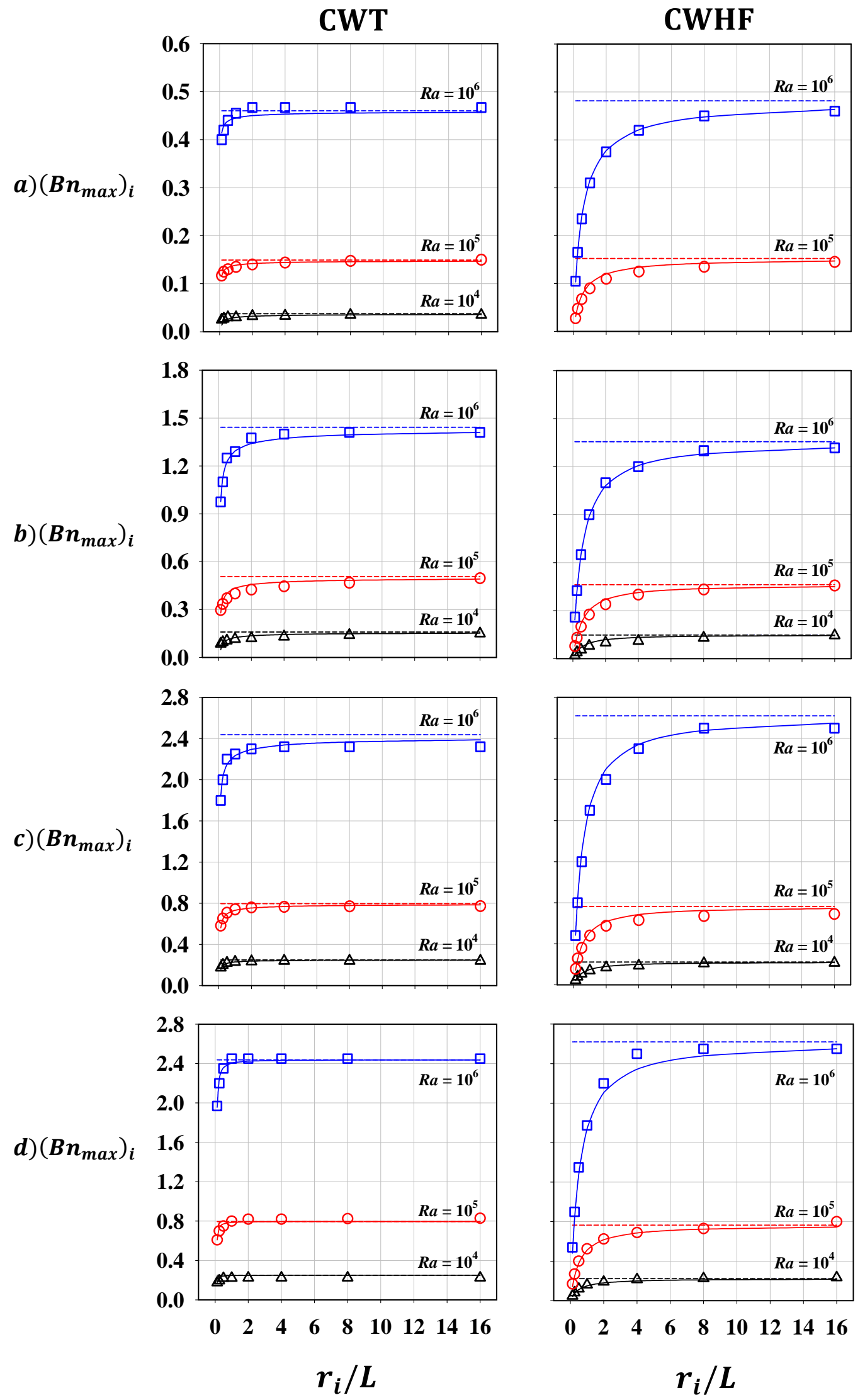

Fig. 12: The variations of $\left(B n_{\max }\right)_{i}$ with $r_{i} / L$ for different values of $R a$ and $A R:$ a) $\left.0.5, \mathrm{~b}\right) 1$, c) 4 , d) 8 at $\operatorname{Pr}=500$ along with the predictions of Eq. (37) and (38) (solid line) for CWT and CWHF respectively. The corresponding values for the $\left(B n_{\max }\right)_{R e c}$ are shown by the dashed line (Eq. (36)). 

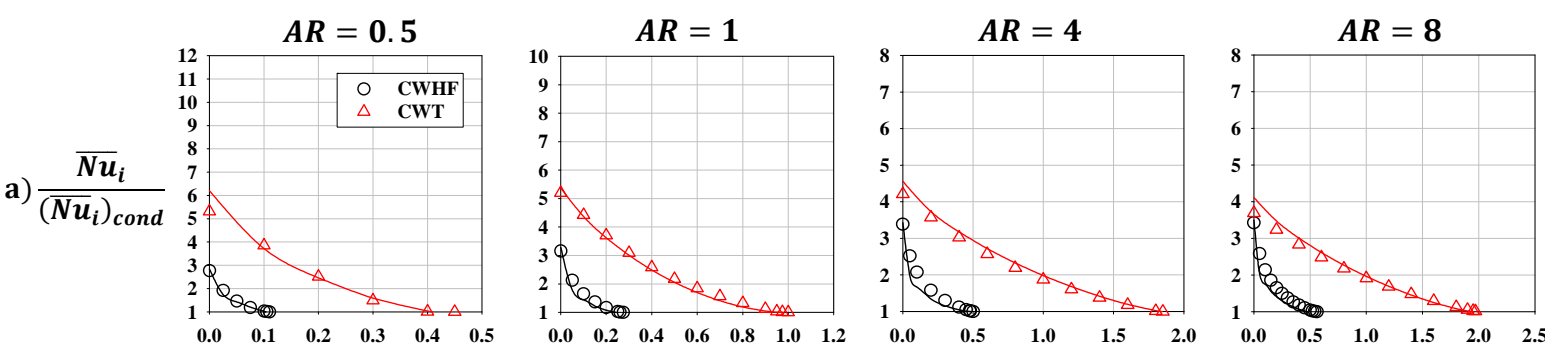

b) $\frac{\overline{\boldsymbol{N u}}_{i}}{\left(\overline{\boldsymbol{N u}}_{i}\right)_{\text {cond }}}$
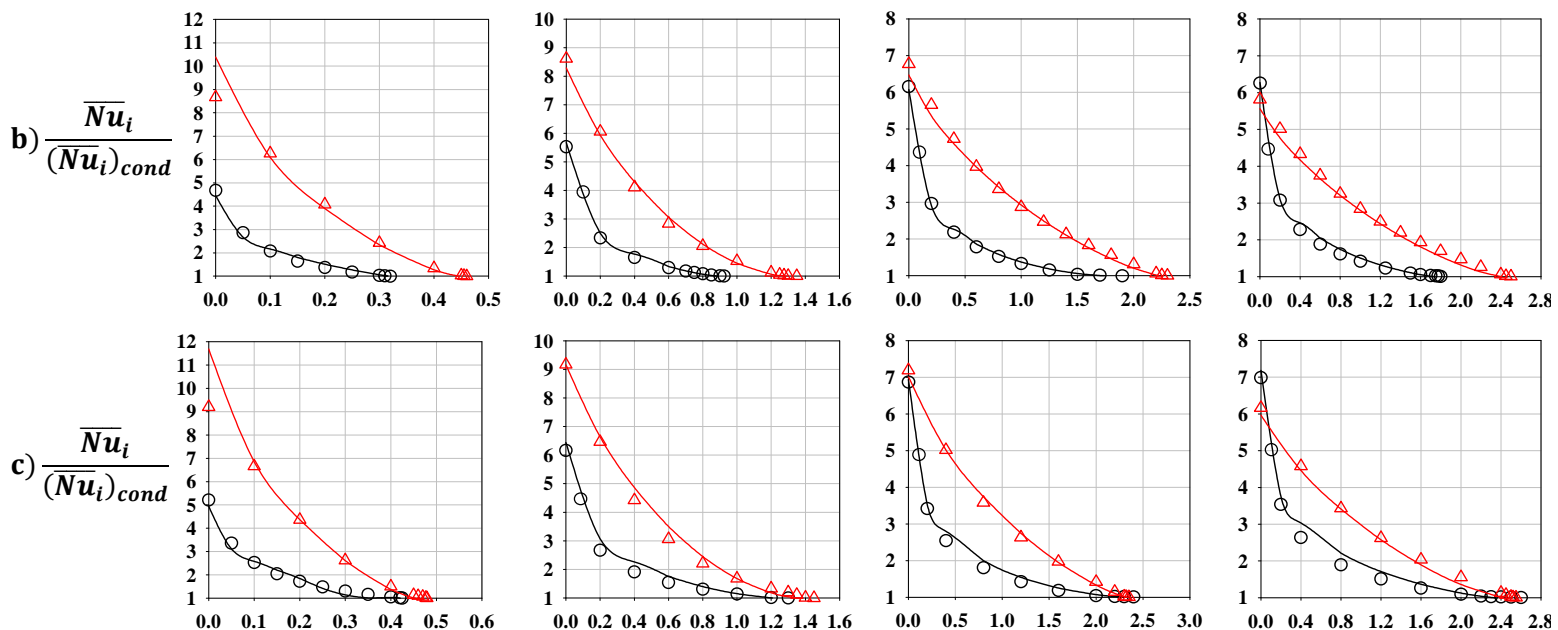

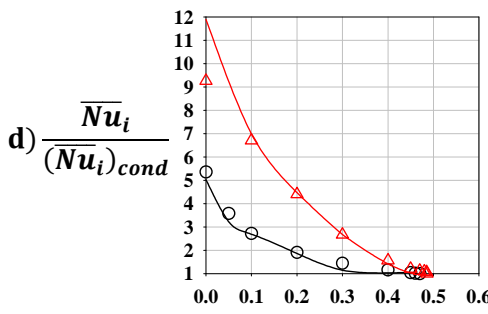

Bn

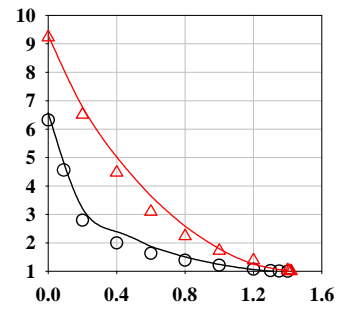

$B n$

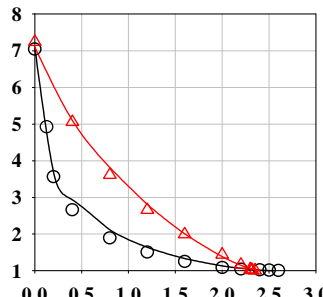

Bn

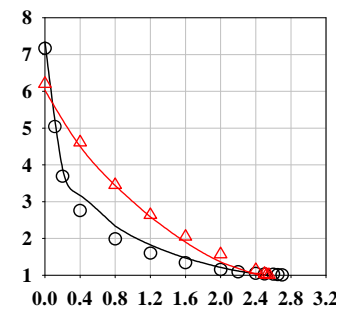

Bn

Fig 13: The variations of $\overline{N u}_{i} /\left(\overline{N u}_{i}\right)_{\text {cond }}$ with $B n$ for different values of $A R$ and $r_{i} / L$ : a) 0.125 , b) 1 , c) 4, d) 16 at $R a=10^{6}$ and $P r=500$ along with predictions of the correlation listed in Table 5 for CWT and CWHF configuration respectively. 